\title{
The interplay between behavior and morphology in the evolutionary dynamics of resource specialization
}

Claus Rueffler (rueffler@zoo.utoronto.ca)

Tom J.M. Van Dooren (t.j.m.van.dooren@biology.leidenuniv.nl)

Johan A.J. Metz (j.a.j.metz@biology.leidenuniv.nl)

\section{Approved by}

Ulf Dieckmann

Program Leader, Evolution and Ecology Program

December 2006 


\section{IIASA STUDIES IN ADAPTIVE DYNAMICS No. 131}

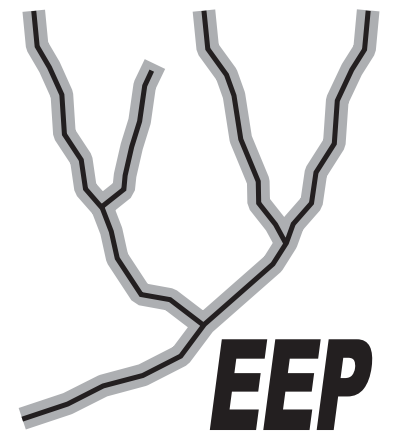

The Evolution and Ecology Program at IIASA fosters the development of new mathematical and conceptual techniques for understanding the evolution of complex adaptive systems.

Focusing on these long-term implications of adaptive processes in systems of limited growth, the Evolution and Ecology Program brings together scientists and institutions from around the world with IIASA acting as the central node.

Scientific progress within the network is collected in the IIASA Studies in Adaptive Dynamics series.
No. 1 Metz JAJ, Geritz SAH, Meszéna G, Jacobs FJA, van Heerwaarden JS: Adaptive Dynamics: A Geometrical Study of the Consequences of Nearly Faithful Reproduction. IIASA Working Paper WP-95-099 (1995). van Strien SJ, Verduyn Lunel SM (eds): Stochastic and Spatial Structures of Dynamical Systems, Proceedings of the Royal Dutch Academy of Science (KNAW Verhandelingen), North Holland, Amsterdam, pp. 183-231 (1996).

No. 2 Dieckmann U, Law R: The Dynamical Theory of Coevolution: A Derivation from Stochastic Ecological Processes. IIASA Working Paper WP-96-001 (1996). Journal of Mathematical Biology 34:579-612 (1996).

No. 3 Dieckmann U, Marrow P, Law R: Evolutionary Cycling of Predator-PreyInteractions: Population Dynamics and the Red Queen. IIASA Preprint (1995). Journal of Theoretical Biology 176:91-102 (1995).

No. 4 Marrow P, Dieckmann U, Law R: Evolutionary Dynamics of Predator-Prey Systems: An Ecological Perspective. IIASA Working Paper WP-96-002 (1996). Journal of Mathematical Biology 34:556-578 (1996).

No. 5 Law R, Marrow P, Dieckmann U: On Evolution under Asymmetric Competition. IIASA Working Paper WP-96-003 (1996). Evolutionary Ecology 11:485-501 (1997).

No. 6 Metz JAJ, Mylius SD, Diekmann O: When Does Evolution Optimize? On the Relation Between Types of Density Dependence and Evolutionarily Stable Life History Parameters. IIASA Working Paper WP-96-004 (1996).

No. 7 Ferrière R, Gatto M: Lyapunov Exponents and the Mathematics of Invasion in Oscillatory or Chaotic Populations. Theoretical Population Biology 48:126-171 (1995).

No. 8 Ferrière R, Fox GA: Chaos and Evolution. IIASA Preprint (1996). Trends in Ecology and Evolution 10:480485 (1995).

No. 9 Ferrière R, Michod RE: The Evolution of Cooperation in Spatially Heterogeneous Populations. IIASA Working Paper WP-96-029 (1996). The American Naturalist 147:692717 (1996).

No. 10 van Dooren TJM, Metz JAJ: Delayed Maturation in Temporally Structured Populations with Non-Equilibrium Dynamics. IIASA Working Paper WP-96-070 (1996). Journal of Evolutionary Biology 11:41-62 (1998).
No. 11 Geritz SAH, Metz JAJ, Kisdi É, Meszéna G: The Dynamics of Adaptation and Evolutionary Branching. IIASA Working Paper WP-96-077 (1996). Physical Review Letters 78:2024-2027 (1997).

No. 12 Geritz SAH, Kisdi É, Meszéna G, Metz JAJ: Evolutionary Singular Strategies and the Adaptive Growth and Branching of the Evolutionary Tree. IIASA Working Paper WP-96-114 (1996). Evolutionary Ecology 12:35-57 (1998).

No. 13 Heino M, Metz JAJ, Kaitala V: Evolution of Mixed Maturation Strategies in Semelparous Life-Histories: The Crucial Role of Dimensionality of Feedback Environment. IIASA Working Paper WP-96-126 (1996). Philosophical Transactions of the Royal Society of London Series B 352:1647-1655 (1997).

No. 14 Dieckmann U: Can Adaptive Dynamics Invade? IIASA Working Paper WP-96-152 (1996). Trends in Ecology and Evolution 12:128-131 (1997).

No. 15 Meszéna G, Czibula I, Geritz SAH: Adaptive Dynamics in a 2-Patch Environment: A Simple Model for Allopatric and Parapatric Speciation. IIASA Interim Report IR-97-001 (1997). Journal of Biological Systems 5:265-284 (1997).

No. 16 Heino M, Metz JAJ, Kaitala V: The Enigma of Frequency-Dependent Selection. IIASA Interim Report IR97-061 (1997). Trends in Ecology and Evolution 13:367-370 (1998).

No. 17 Heino M: Management of Evolving Fish Stocks. IIASA Interim Report IR-97-062 (1997). Canadian Journal of Fisheries and Aquatic Sciences 55:1971-1982 (1998).

No. 18 Heino M: Evolution of Mixed Reproductive Strategies in Simple Life-History Models. IIASA Interim Report IR-97063 (1997).

No. 19 Geritz SAH, van der Meijden E, Metz JAJ: Evolutionary Dynamics of Seed Size and Seedling Competitive Ability. IIASA Interim Report IR-97-071 (1997). Theoretical Population Biology 55:324-343 (1999).

No. 20 Galis F, Metz JAJ: Why Are There So Many Cichlid Species? On the Interplay of Speciation and Adaptive Radiation. IIASA Interim Report IR-97-072 (1997). Trends in Ecology and Evolution 13:1-2 (1998). 
No. 21 Boerlijst MC, Nowak MA, Sigmund K: Equal Pay for all Prisoners/ The Logic of Contrition. IIASA Interim Report IR-97-073 (1997). American Mathematical Society Monthly 104:303-307 (1997). Journal of Theoretical Biology 185:281-293 (1997).

No. 22 Law R, Dieckmann U: Symbiosis Without Mutualism and the Merger of Lineages in Evolution. IIASA Interim Report IR-97-074 (1997). Proceedings of the Royal Society of London Series B 265:1245-1253 (1998).

No. 23 Klinkhamer PGL, de Jong TJ, Metz JAJ: Sex and Size in Cosexual Plants. IIASA Interim Report IR-97-078 (1997). Trends in Ecology and Evolution 12:260-265 (1997).

No. 24 Fontana W, Schuster P: Shaping Space: The Possible and the Attainable in RNA Genotype-Phenotype Mapping. IIASA Interim Report IR-98-004 (1998). Journal of Theoretical Biology 194:491-515 (1998).

No. 25 Kisdi É, Geritz SAH: Adaptive Dynamics in Allele Space: Evolution of Genetic Polymorphism by Small Mutations in a Heterogeneous Environment. IIASA Interim Report IR-98-038 (1998). Evolution 53:993-1008 (1999).

No. 26 Fontana W, Schuster P: Continuity in Evolution: On the Nature of Transitions. IIASA Interim Report IR-98-039 (1998). Science 280:1451-1455 (1998).

No. 27 Nowak MA, Sigmund K: Evolution of Indirect Reciprocity by Image Scoring/ The Dynamics of Indirect Reciprocity. IIASA Interim Report IR-98-040 (1998). Nature 393:573-577 (1998). Journal of Theoretical Biology 194:561574 (1998).

No. 28 Kisdi É: Evolutionary Branching Under Asymmetric Competition. IIASA Interim Report IR-98-045 (1998). Journal of Theoretical Biology 197:149-162 (1999).

No. 29 Berger U: Best Response Adaptation for Role Games. IIASA Interim Report IR-98-086 (1998).

No. 30 van Dooren TJM: The Evolutionary Ecology of Dominance-Recessivity. IIASA Interim Report IR-98-096 (1998). Journal of Theoretical Biology 198:519-532 (1999).

No. 31 Dieckmann U, O'Hara B, Weisser W: The Evolutionary Ecology of Dispersal. IIASA Interim Report IR-98-108 (1998). Trends in Ecology and Evolution 14:88-90 (1999).

No. 32 Sigmund K: Complex Adaptive Systems and the Evolution of Reciprocation. IIASA Interim Report IR-98-100 (1998). Ecosystems 1:444-448 (1998).

No. 33 Posch M, Pichler A, Sigmund K: The Efficiency of Adapting Aspiration Levels. IIASA Interim Report IR-98103 (1998). Proceedings of the Royal Society London Series B 266:1427-1435 (1999).

No. 34 Mathias A, Kisdi É: Evolutionary Branching and Coexistence of Germination Strategies. IIASA Interim Report IR-99-014 (1999).

No. 35 Dieckmann U, Doebeli M: On the Origin of Species by Sympatric Speciation. IIASA Interim Report IR-99-013 (1999). Nature 400:354-357 (1999).

No. 36 Metz JAJ, Gyllenberg M: How Should We Define Fitness in Structured Metapopulation Models? Including an Application to the Calculation of Evolutionarily Stable Dispersal Strategies. IIASA Interim Report IR-99-019 (1999). Proceedings of the Royal Society of London Series B 268:499508 (2001)
No. 37 Gyllenberg M, Metz JAJ: On Fitness in Structured Metapopulations. IIASA Interim Report IR-99-037 (1999). Journal of Mathematical Biology 43:545-560 (2001).

No. 38 Meszéna G, Metz JAJ: Species Diversity and Population Regulation: The Importance of Environmental Feedback Dimensionality. IIASA Interim Report IR-99-045 (1999).

No. 39 Kisdi É, Geritz SAH: Evolutionary Branching and Sympatric Speciation in Diploid Populations. IIASA Interim Report IR-99-048 (1999).

No. 40 Ylikarjula J, Heino M, Dieckmann U: Ecology and Adaptation of Stunted Growth in Fish. IIASA Interim Report IR-99-050 (1999). Evolutionary Ecology 13:433-453 (1999).

No. 41 Nowak MA, Sigmund K: Games on Grids. IIASA Interim Report IR-99-038 (1999). Dieckmann U, Law R, Metz JAJ (eds): The Geometry of Ecological Interactions: Simplifying Spatial Complexity, Cambridge University Press, Cambridge, UK, pp. 135-150 (2000).

No. 42 Ferrière R, Michod RE: Wave Patterns in Spatial Games and the Evolution of Cooperation. IIASA Interim Report IR-99-041 (1999). Dieckmann U, Law R, Metz JAJ (eds): The Geometry of Ecological Interactions: Simplifying Spatial Complexity, Cambridge University Press, Cambridge, UK, pp. 318-332 (2000).

No. 43 Kisdi É, Jacobs FJA, Geritz SAH: Red Queen Evolution by Cycles of Evolutionary Branching and Extinction. IIASA Interim Report IR-00-030 (2000). Selection 2:161$176(2001)$.

No. 44 Meszéna G, Kisdi É, Dieckmann U, Geritz SAH, Metz JAJ: Evolutionary Optimisation Models and Matrix Games in the Unified Perspective of Adaptive Dynamics. IIASA Interim Report IR-00-039 (2000). Selection 2:193-210 (2001).

No. 45 Parvinen K, Dieckmann U, Gyllenberg M, Metz JAJ: Evolution of Dispersal in Metapopulations with Local Density Dependence and Demographic Stochasticity. IIASA Interim Report IR-00-035 (2000). Journal of Evolutionary Biology 16:143-153 (2003).

No. 46 Doebeli M, Dieckmann U: Evolutionary Branching and Sympatric Speciation Caused by Different Types of Ecological Interactions. IIASA Interim Report IR-00-040 (2000). The American Naturalist 156:S77-S101 (2000).

No. 47 Heino M, Hanski I: Evolution of Migration Rate in a Spatially Realistic Metapopulation Model. IIASA Interim Report IR-00-044 (2000). The American Naturalist 157:495$511(2001)$.

No. 48 Gyllenberg M, Parvinen K, Dieckmann U: Evolutionary Suicide and Evolution of Dispersal in Structured Metapopulations. IIASA Interim Report IR-00-056 (2000). Journal of Mathematical Biology 45:79-105 (2002).

No. 49 van Dooren TJM: The Evolutionary Dynamics of Direct Phenotypic Overdominance: Emergence Possible, Loss Probable. IIASA Interim Report IR-00-048 (2000). Evolution 54:1899-1914 (2000).

No. 50 Nowak MA, Page KM, Sigmund K: Fairness Versus Reason in the Ultimatum Game. IIASA Interim Report IR00-57 (2000). Science 289:1773-1775 (2000).

No. 51 de Feo O, Ferrière R: Bifurcation Analysis of Population Invasion: On-Off Intermittency and Basin Riddling. IIASA Interim Report IR-00-074 (2000). International Journal of Bifurcation and Chaos 10:443-452 (2000). 
No. 52 Heino M, Laaka-Lindberg S: Clonal Dynamics and Evolution of Dormancy in the Leafy Hepatic Lophozia Silvicola. IIASA Interim Report IR-01-018 (2001). Oikos 94:525-532 (2001).

No. 53 Sigmund K, Hauert C, Nowak MA: Reward and Punishment in Minigames. IIASA Interim Report IR-01-031 (2001). Proceedings of the National Academy of Sciences of the USA 98:10757-10762 (2001).

No. 54 Hauert C, De Monte S, Sigmund K, Hofbauer J: Oscillations in Optional Public Good Games. IIASA Interim Report IR-01-036 (2001).

No. 55 Ferrière R, Le Galliard J: Invasion Fitness and Adaptive Dynamics in Spatial Population Models. IIASA Interim Report IR-01-043 (2001). Clobert J, Dhondt A, Danchin E, Nichols J (eds): Dispersal, Oxford University Press, pp. 57-79 (2001).

No. 56 de Mazancourt C, Loreau M, Dieckmann U: Can the Evolution of Plant Defense Lead to Plant-Herbivore Mutualism? IIASA Interim Report IR-01-053 (2001). The American Naturalist 158:109-123 (2001).

No. 57 Claessen D, Dieckmann U: Ontogenetic Niche Shifts and Evolutionary Branching in Size-Structured Populations. IIASA Interim Report IR-01-056 (2001). Evolutionary Ecology Research 4:189-217 (2002).

No. 58 Brandt H: Correlation Analysis of Fitness Landscapes. IIASA Interim Report IR-01-058 (2001).

No. 59 Dieckmann U: Adaptive Dynamics of Pathogen-Host Interacations. IIASA Interim Report IR-02-007 (2002). Dieckmann U, Metz JAJ, Sabelis MW, Sigmund K (eds): Adaptive Dynamics of Infectious Diseases: In Pursuit of Virulence Management, Cambridge University Press, Cambridge, UK, pp. 39-59 (2002).

No. 60 Nowak MA, Sigmund K: Super- and Coinfection: The Two Extremes. IIASA Interim Report IR-02-008 (2002). Dieckmann U, Metz JAJ, Sabelis MW, Sigmund K (eds): Adaptive Dynamics of Infectious Diseases: In Pursuit of Virulence Management, Cambridge University Press, Cambridge, UK, pp. 124-137 (2002).

No. 61 Sabelis MW, Metz JAJ: Evolution Management: Taking Stock - Relating Theory to Experiment. IIASA Interim Report IR-02-009 (2002). Dieckmann U, Metz JAJ, Sabelis MW, Sigmund K (eds): Adaptive Dynamics of Infectious Diseases: In Pursuit of Virulence Management, Cambridge University Press, Cambridge, UK, pp. 379-398 (2002).

No. 62 Cheptou P, Dieckmann U: The Evolution of SelfFertilization in Density-Regulated Populations . IIASA Interim Report IR-02-024 (2002). Proceedings of the Royal Society of London Series B 269:1177-1186(2002).

No. 63 Bürger R: Additive Genetic Variation Under Intraspecific Competition and Stabilizing Selection: A Two-Locus Study. IIASA Interim Report IR-02-013 (2002). Theoretical Population Biology 61:197-213 (2002).

No. 64 Hauert C, De Monte S, Hofbauer J, Sigmund K: Volunteering as Red Queen Mechanism for Co-operation in Public Goods Games. IIASA Interim Report IR-02-041 (2002). Science 296:1129-1132 (2002).

No. 65 Dercole F, Ferrière R, Rinaldi S: Ecological Bistability and Evolutionary Reversals under Asymmetrical Competition. IIASA Interim Report IR-02-053 (2002). Evolution 56:1081-1090 (2002).
No. 66 Dercole F, Rinaldi S: Evolution of Cannibalistic Traits: Scenarios Derived from Adaptive Dynamics. IIASA Interim Report IR-02-054 (2002). Theoretical Population Biology 62:365-374 (2002).

No. 67 Bürger R, Gimelfarb A: Fluctuating Environments and the Role of Mutation in Maintaining Quantitative Genetic Variation. IIASA Interim Report IR-02-058 (2002). Genetical Research 80:31-46 (2002).

No. 68 Bürger R: On a Genetic Model of Intraspecific Competition and Stabilizing Selection. IIASA Interim Report IR02-062 (2002). Amer. Natur. 160:661-682 (2002).

No. 69 Doebeli M, Dieckmann U: Speciation Along Environmental Gradients. IIASA Interim Report IR-02-079 (2002). Nature 421:259-264 (2003).

No. 70 Dercole F, Irisson J, Rinaldi S: Bifurcation Analysis of a Prey-Predator Coevolution Model. IIASA Interim Report IR-02-078 (2002). SIAM Journal on Applied Mathematics 63:1378-1391 (2003).

No. 71 Le Galliard J, Ferrière R, Dieckmann U: The Adaptive Dynamics of Altruism in Spatially Heterogeneous Populations. IIASA Interim Report IR-03-006 (2003). Evolution 57:1-17 (2003).

No. 72 Taborsky B, Dieckmann U, Heino M: Unexpected Discontinuities in Life-History Evolution under SizeDependent Mortality. IIASA Interim Report IR-03-004 (2003). Proceedings of the Royal Society of London Series B 270:713-721 (2003).

No. 73 Gardmark A, Dieckmann U, Lundberg P: LifeHistory Evolution in Harvested Populations: The Role of Natural Predation. IIASA Interim Report IR-03-008 (2003). Evolutionary Ecology Research 5:239-257 (2003).

No. 74 Mizera F, Meszéna G: Spatial Niche Packing, Character Displacement and Adaptive Speciation Along an Environmental Gradient. IIASA Interim Report IR-03-062 (2003). Evolutionary Ecology Research 5:363-382 (2003).

No. 75 Dercole F: Remarks on Branching-Extinction Evolutionary Cycles. IIASA Interim Report IR-03-077 (2003). Journal of Mathematical Biology 47:569-580 (2003).

No. 76 Hofbauer J, Sigmund K: Evolutionary Game Dynamics. IIASA Interim Report IR-03-078 (2003). Bulletin of the American Mathematical Society 40:479-519 (2003).

No. 77 Ernande B, Dieckmann U, Heino M: Adaptive Changes in Harvested Populations: Plasticity and Evolution of Age and Size at Maturation. IIASA Interim Report IR03-058 (2003). Proceedings of the Royal Society of London Series B-Biological Sciences 271:415-423 (2004).

No. 78 Hanski I, Heino M: Metapopulation-Level Adaptation of Insect Host Plant Preference and Extinction-Colonization Dynamics in Heterogeneous Landscapes. IIASA Interim Report IR-03-028 (2003). Theoretical Population Biology 63:309-338 (2003).

No. 79 van Doorn G, Dieckmann U, Weissing FJ: Sympatric Speciation by Sexual Selection: A Critical Re-Evaluation. IIASA Interim Report IR-04-003 (2004). American Naturalist 163:709-725 (2004).

No. 80 Egas M, Dieckmann U, Sabelis MW: Evolution Restricts the Coexistence of Specialists and Generalists - the Role of Trade-off Structure. IIASA Interim Report IR-04-004 (2004). American Naturalist 163:518-531 (2004). 
No. 81 Ernande B, Dieckmann U: The Evolution of Phenotypic Plasticity in Spatially Structured Environments: Implications of Intraspecific Competition, Plasticity Costs, and Environmental Characteristics. IIASA Interim Report IR-04-006 (2004). Journal of Evolutionary Biology 17:613-628 (2004).

No. 82 Cressman R, Hofbauer J: Measure Dynamics on a One-Dimensional Continuous Trait Space: Theoretical Foundations for Adaptive Dynamics. IIASA Interim Report IR04-016 (2004).

No. 83 Cressman R: Dynamic Stability of the Replicator Equation with Continuous Strategy Space. IIASA Interim Report IR-04-017 (2004).

No. 84 Ravigné V, Olivieri I, Dieckmann U: Implications of Habitat Choice for Protected Polymorphisms. IIASA Interim Report IR-04-005 (2004). Evolutionary Ecology Research 6:125-145 (2004).

No. 85 Nowak MA, Sigmund K: Evolutionary Dynamics of Biological Games. IIASA Interim Report IR-04-013 (2004). Science 303:793-799 (2004).

No. 86 Vukics A, Asbóth J, Meszéna G: Speciation in Multidimensional Evolutionary Space. IIASA Interim Report IR-04-028 (2004). Physical Review 68:041-903 (2003).

No. 87 de Mazancourt C, Dieckmann U: Trade-off Geometries and Frequency-dependent Selection. IIASA Interim Report IR-04-039 (2004). American Naturalist 164:765-778 (2004).

No. 88 Cadet CR, Metz JAJ, Klinkhamer PGL: Size and the Not-So-Single Sex: Disentangling the Effects of Size on Sex Allocation. IIASA Interim Report IR-04-084 (2004). American Naturalist 164:779-792 (2004).

No. 89 Rueffler C, van Dooren TJM, Metz JAJ: Adaptive Walks on Changing Landscapes: Levins' Approach Extended. IIASA Interim Report IR-04-083 (2004). Theoretical Population Biology 65:165-178 (2004).

No. 90 de Mazancourt C, Loreau M, Dieckmann U: Understanding Mutualism When There is Adaptation to the Partner. IIASA Interim Report IR-05-016 (2005). Journal of Ecology 93:305-314 (2005).

No. 91 Dieckmann U, Doebeli M: Pluralism in Evolutionary Theory. IIASA Interim Report IR-05-017 (2005). Journal of Evolutionary Biology 18:1209-1213 (2005).

No. 92 Doebeli M, Dieckmann U, Metz JAJ, Tautz D: What We Have Also Learned: Adaptive Speciation is Theoretically Plausible. IIASA Interim Report IR-05-018 (2005). Evolution 59:691-695 (2005).

No. 93 Egas M, Sabelis MW, Dieckmann U: Evolution of Specialization and Ecological Character Displacement of Herbivores Along a Gradient of Plant Quality. IIASA Interim Report IR-05-019 (2005). Evolution 59:507-520 (2005).

No. 94 Le Galliard J, Ferrière R, Dieckmann U: Adaptive Evolution of Social Traits: Origin, Trajectories, and Correlations of Altruism and Mobility. IIASA Interim Report IR05-020 (2005). American Naturalist 165:206-224 (2005).

No. 95 Doebeli M, Dieckmann U: Adaptive Dynamics as a Mathematical Tool for Studying the Ecology of Speciation Processes. IIASA Interim Report IR-05-022 (2005). Journal of Evolutionary Biology 18:1194-1200 (2005).

No. 96 Brandt H, Sigmund K: The Logic of Reprobation: Assessment and Action Rules for Indirect Reciprocity. IIASA Interim Report IR-04-085 (2004). Journal of Theoretical Biology 231:475-486 (2004).
No. 97 Hauert C, Haiden N, Sigmund K: The Dynamics of Public Goods. IIASA Interim Report IR-04-086 (2004). Discrete and Continuous Dynamical Systems - Series B 4:575587 (2004).

No. 98 Meszéna G, Gyllenberg M, Jacobs FJA, Metz JAJ: Link Between Population Dynamics and Dynamics of Darwinian Evolution. IIASA Interim Report IR-05-026 (2005). Physical Review Letters 95:Article 078105 (2005).

No. 99 Meszéna G: Adaptive Dynamics: The Continuity Argument. IIASA Interim Report IR-05-032 (2005).

No. 100 Brännström NA, Dieckmann U: Evolutionary Dynamics of Altruism and Cheating Among Social Amoebas. IIASA Interim Report IR-05-039 (2005). Proceedings of the Royal Society London Series B 272:1609-1616 (2005).

No. 101 Meszéna G, Gyllenberg M, Pasztor L, Metz JAJ: Competitive Exclusion and Limiting Similarity: A Unified Theory. IIASA Interim Report IR-05-040 (2005).

No. 102 Szabo P, Meszéna G: Limiting Similarity Revisited. IIASA Interim Report IR-05-050 (2005).

No. 103 Krakauer DC, Sasaki A: The Greater than Two-Fold Cost of Integration for Retroviruses. IIASA Interim Report IR-05-069 (2005).

No. 104 Metz JAJ: Eight Personal Rules for Doing Science. IIASA Interim Report IR-05-073 (2005). Journal of Evolutionary Biology 18:1178-1181 (2005).

No. 105 Beltman JB, Metz JAJ: Speciation: More Likely Through a Genetic or Through a Learned Habitat Preference? IIASA Interim Report IR-05-072 (2005). Proceedings of the Royal Society of London Series B 272:1455-1463 (2005).

No. 106 Durinx M, Metz JAJ: Multi-type Branching Processes and Adaptive Dynamics of Structured Populations. IIASA Interim Report IR-05-074 (2005). Haccou P, Jager P, Vatutin V (eds): Branching Processes: Variation, Growth and Extinction of Populations, Cambridge University Press, Cambridge, UK, pp. 266-278 (2005).

No. 107 Brandt H, Sigmund K: The Good, the Bad and the Discriminator - Errors in Direct and Indirect Reciprocity. IIASA Interim Report IR-05-070 (2005). Journal of Theoretical Biology 239:183-194 (2006).

No. 108 Brandt H, Hauert C, Sigmund K: Punishing and Abstaining for Public Goods. IIASA Interim Report IR-05-071 (2005). Proceedings of the National Academy of Sciences of the United States of America 103:495-497 (2006).

No. 109 Ohtsuki A, Sasaki A: Epidemiology and DiseaseControl Under Gene-for-Gene Plant-Pathogen Interaction. IIASA Interim Report IR-05-068 (2005).

No. 110 Brandt H, Sigmund K: Indirect Reciprocity, ImageScoring, and Moral Hazard. IIASA Interim Report IR-05078 (2005). Proceedings of the National Academy of Sciences of the United States of America 102:2666-2670 (2005).

No. 111 Nowak MA, Sigmund K: Evolution of Indirect Reciprocity. IIASA Interim Report IR-05-079 (2005). Nature 437:1292-1298 (2005).

No. 112 Kamo M, Sasaki A: Evolution Towards Multi-Year Periodicity in Epidemics. IIASA Interim Report IR-05-080 (2005). Ecology Letters 8:378-385 (2005). 
No. 113 Dercole F, Ferrière R, Gragnani A, Rinaldi S: Coevolution of Slow-fast Populations: Evolutionary Sliding, Evolutionoary Pseudo-equilibria, and Complex Red Queen Dynamics. IIASA Interim Report IR-06-006 (2006). Proceedings of the Royal Society B-Biological Sciences 273:983-990 (2006).

No. 114 Dercole F: Border Collision Bifurcations in the Evolution of Mutualistic Interactions. IIASA Interim Report IR-05-083 (2005). International Journal of Bifurcation and Chaos 15:2179-2190 (2005).

No. 115 Dieckmann U, Heino M, Parvinen K: The Adaptive Dynamics of Function-Valued Traits. IIASA Interim Report IR-06-036 (2006). Journal of Theoretical Biology 241:370389 (2006).

No. 116 Dieckmann U, Metz JAJ: Surprising Evolutionary Predictions from Enhanced Ecological Realism. IIASA Interim Report IR-06-037 (2006). Theoretical Population Biology 69:263-281 (2006).

No. 117 Dieckmann U, Brännström NA, HilleRisLambers R, Ito H: The Adaptive Dynamics of Community Structure. IIASA Interim Report IR-06-038 (2006). Takeuchi Y, Iwasa Y, Sato K (eds): Mathematics for Ecology and Environmental Sciences, Springer, Berlin Heidelberg, pp. 145-177 (2007).

No. 118 Gardmark A, Dieckmann U: Disparate Maturation Adaptations to Size-dependent Mortality. IIASA Interim Report IR-06-039 (2006). Proceedings of the Royal Society London Series B 273:2185-2192 (2006).

No. 119 van Doorn G, Dieckmann U: The Long-term Evolution of Multi-locus Traits Under Frequency-dependent Disruptive Selection. IIASA Interim Report IR-06-041 (2006). Evolution 60:2226-2238 (2006).

No. 120 Doebeli M, Blok HJ, Leimar O, Dieckmann U: Multimodal Pattern Formation in Phenotype Distributions of Sexual Populations. IIASA Interim Report IR-06-046 (2006). Proceedings of the Royal Society London Series B 274:347357 (2007).

No. 121 Dunlop ES, Shuter BJ, Dieckmann U: The Demographic and Evolutionary Consequences of Selective Mortality: Predictions from an Eco-genetic Model of the Smallmouth Bass. IIASA Interim Report IR-06-060 (2006).
No. 122 Metz JAJ: Fitness. IIASA Interim Report IR-06061 (2006).

No. 123 Brandt H, Ohtsuki H, Iwasa Y, Sigmund K: A Survey on Indirect Reciprocity. IIASA Interim Report IR-06-065 (2006). Takeuchi Y, Iwasa Y, Sato K (eds): Mathematics for Ecology and Environmental Sciences, Springer, Berlin Heidelberg, pp. 21-51 (2007).

No. 124 Dercole F, Loiacono D, Rinaldi S: Synchronization in Ecological Networks: A Byproduct of Darwinian Evolution? IIASA Interim Report IR-06-068 (2006).

No. 125 Dercole F, Dieckmann U, Obersteiner M, Rinaldi S: Adaptive Dynamics and Technological Change. IIASA Interim Report IR-06-070 (2006).

No. 126 Rueffler C, van Dooren TJM, Metz JAJ: The Evolution of Resource Specialization Through FrequencyDependent and Frequency-Independent Mechanisms. IIASA Interim Report IR-06-073 (2006). American Naturalist 167:81-93 (2006).

No. 127 Rueffler C, Egas M, Metz JAJ: Evolutionary Predictions Should be Based on Individual Traits. IIASA Interim Report IR-06-074 (2006). American Naturalist 168:148-162 (2006).

No. 128 Kamo M, Sasaki A, Boots M: The Role of Trade-Off Shapes in the Evolution of Virulence in Spatial Host-Parasite Interactions: An Approximate Analytical Approach. IIASA Interim Report IR-06-075 (2006).

No. 129 Boots M, Kamo M, Sasaki A: The Implications of Spatial Structure Within Populations to the Evolution of Parasites . IIASA Interim Report IR-06-078 (2006).

No. 130 Andreasen V, Sasaki A: Shaping the Phylogenetic Tree of Influenza by Cross-Immunity. IIASA Interim Report IR-06-079 (2006).

No. 131 Rueffler C, van Dooren TJM, Metz JAJ: The Interplay Between Behavior and Morphology in the Evolutionary Dynamics of Resource Specialization. IIASA Interim Report IR-06-082 (2006).

Issues of the IIASA Studies in Adaptive Dynamics series can be obtained at www.iiasa.ac.at/Research/EEP/Series.html or by writing to eep@iiasa.ac.at. 


\section{Contents}

Abstract. 


\title{
The interplay between behavior and morphology in the evolutionary dynamics of resource specialization
}

\author{
Claus Rueffler ${ }^{1}$, Tom J.M. Van Dooren ${ }^{2}$, and Johan A. J. Metz ${ }^{3,4}$ \\ Institute of Biology Leiden, Leiden University, Kaiserstraat 63, 2311 GP Leiden, The Netherlands
}

\begin{abstract}
We analyze the consequences of diet choice behavior for the evolutionary dynamics of foraging traits by means of a mathematical model. The model is characterized by the following features: Consumers feed on two different substitutable resources that are distributed in a fine grained manner. Consumers decide upon encounter with a resource item whether to attack it or not so as to maximize their energy intake. Simultaneously, evolution occurs in either of five different traits involved in the foraging process. For each trait, a trade-off exists between the consumer's ability to forage on the alternative resources. The model predicts that flexible diet choice behavior can guide the direction of evolutionary change and that flexible behavior can mediate coexistence of different consumer types. Such polymorphisms can evolve from a monomorphic population at evolutionary branching points and also at points where a small genetic change in a trait can provoke a sharp instantaneous and non-genetic change in choice behavior. In the case of weak trade-offs, the evolutionary dynamics of a dimorphic consumer population can lead to alternative evolutionarily stable communities. The robustness of these predictions is checked with individual-based simulations and by relaxing the assumption of optimally foraging consumers.
\end{abstract}

Subject headings: adaptive dynamics, coexistence, generalist, optimal diet choice, polymorphism, specialist, trade-off

\footnotetext{
${ }^{1}$ corresponding author, present address: Department of Ecology and Evolutionary Biology, University of Toronto 25 Harbord St., Toronto, Ontario, M5S 3G5, Canada, rueffler@zoo.utoronto.ca

${ }^{2}$ t.j.m.van.dooren@biology.leidenuniv.nl

3 j.a.j.metz@biology.leidenuniv.nl

${ }^{4}$ Evolution and Ecology Program, International Institute of Applied Systems Analysis, A-2361 Laxenburg, Austria
} 


\section{Introduction}

The fitness of an individual depends on traits that change relatively little during a lifetime and on behavioral patterns that can change on much faster time scales. Waddington pointed out that behavioral patterns can direct the evolution of less labile traits by influencing the strength and direction of selection pressures acting on other aspects of the phenotype (e.g. Waddington 1975; for reviews see Bateson 1988, Plotkin 1988, Wcislo 1989). This paper focuses on the particular case where dietary choice behavior interacts with the evolution of genetically determined foraging traits of a consumer feeding on multiple resources. To illustrate this interaction we might think of a seed-eating bird species. Selection acts on the bill such that its success in extracting seeds would be maximized and the handling time to do so would be minimized. The strength and direction of selection is determined by the behavioral diet choice of the individual: If birds accept a variety of different seeds, the bill will form a compromise imposed by conflicting needs. A bill suitable for foraging on small seeds is most likely unsuitable for foraging on large seeds and if both types are included in the diet, this will result in a generalist bill. If a bird solely chooses small seeds, selection will favor all morphological adaptations that increase the foraging rate for them, irrespectively of how detrimental such adaptations are on the birds foraging rate for large seeds. This example illustrates how diet choice behavior sets the selection pressure for morphological characteristics. On the other hand, diet choice itself is influenced by the morphological traits of the bird, because these traits determine the behavior by which an individual can, given some time constraint, maximize its energy uptake (Stephens and Krebs 1986). An additional complexity arises when consumers regulate the abundance of their resources. In this case morphological traits and diet choice behavior affect the abundance of resources while, in turn, resource availability determines diet choice behavior and thereby influences the selection pressures acting on morphological traits.

The main goal of this paper is to show how behavior can interact with the evolutionary dynamics of morphological traits. As a case in point, the evolution of foraging traits of one consumer species feeding on two different resources is studied. This situation has been analyzed in detail by Rueffler et al. (2006a), in a study that built further on a series of models by Lawlor and Maynard Smith (1976), Lundberg and Stenseth (1985), Abrams (1986) and Wilson and Turelli (1986). In all these models it is assumed that consumers behave opportunistically, i.e., they attack any prey they encounter, regardless of their morphology. Under this assumption the evolutionary dynamics can be classified with respect to two independent properties: the shape of the trade-off curve and the possibility for selection to be frequency-dependent (Rueffler et al. 2006a). Trade-off shape is the main determinant of evolution in the frequency-independent case, as introduced by Levins (1962). The curvature of the trade-off determines whether the generalist trait is an endpoint of evolution, or whether it is susceptible to invasion by deviating mutants. Frequency-dependence, on the other hand, determines whether it is possible for different types to coexist in a protected polymorphism and whether such polymorphisms can emerge at an evolutionary branching point through a series of mutational steps of small effect (Metz et al. 1996; Geritz et al. 1998). In Rueffler et al. (2006a) we show that the presence or absence of frequency-dependent selection depends on the trait that 
is considered to be evolutionarily variable.

In this paper the constraint of opportunistic consumer behavior is removed and the composition of a consumer's diet is derived from optimal diet theory (Stephens and Krebs 1986). The approach is roughly as follows. The first step is to determine the optimal behavior of residents in a morphologically monomorphic population. Subsequently, a mutant type is envisaged that differs in its morphology from the resident population. The behavior of mutant individuals depends on their own morphology and on the abundance of the resources, as determined by the resident type. The joint effects of morphology and behavior decide whether the mutant is going to be successful or will disappear from the population. Given successful invasion of an initially rare mutant several different configurations occur in practice for the new population dynamical equilibrium. Firstly, the mutant replaces the former resident and shows the same diet choice as its predecessor. Secondly, the mutant replaces the former resident and shows a diet choice different from that of its predecessor. Thirdly, the mutant coexists with the former resident in a protected dimorphism where both types choose the same resources as the former resident; and fourthly, the mutant coexists with the former resident in a protected dimorphism in which the former resident sticks to its behavior while the mutant adopts a behavior different from that of the former resident. By following a series of consecutive mutation events the dynamic interplay between instantaneously optimized behavior and the evolutionary dynamics of morphological traits can be studied.

In order to distinguish between a consumer's ability to use different resources and a consumers choice behavior with respect to different resources, the terminology of Rosenzweig $(1981,1987)$ will be adopted here. A consumer that is equally well adapted to utilize a variety of resources is called a "generalist" while a consumer that is specialized in its abilities on a few resources at the cost of being poorly adapted on other resources is called a "specialist". These terms refer to genetically determined foraging traits, whatever their exact nature, that are assumed to change on an evolutionary time scale through the invasion of novel mutants. To simplify matters, in the remainder of this paper we refer to traits of this category as morphological. At the behavioral end, a consumer attacking all encountered prey is called "opportunist" while a choosy consumer is called "selector". These terms refer to diet choice behavior, which is assumed here to be adjusted instantaneously so as to maximize energy intake. We will refer to traits of this second category as behavioral.

The structure of the present paper is as follows. First a model of one opportunistic consumer feeding on two discrete resources is introduced, based on Rueffler et al. (2006a), which is subsequently extended towards flexible consumer behavior. The resulting evolutionary dynamics of the model with flexible diet choice are compared to those obtained in Rueffler et al. (2006a) and checked with individual-based simulations. Finally, the assumption that consumers behave optimally is dropped by introducing foraging inaccuracy. 


\section{The model}

In this section a population dynamical model of one consumer feeding on two resources is constructed. The resources are assumed to be nutritionally substitutable and homogeneously distributed in space. This model is identical to the one presented in Rueffler et al. (2006a), where more details of the model description can be found. Based on the population dynamics of the consumer an expression for invasion fitness is derived that is used to study both the dynamics of morphological traits and behavior. Table 1 gives an overview of all model parameters.

\section{Population dynamics}

The derivation of the population dynamical model is based on a time scale argument (Schoener 1978; Schaffer 1981; Geritz and Kisdi 2004). The consumer population has non-overlapping generations and reproduces once per year. The population is censused after juvenile mortality and consumer densities are assumed to stay constant until the next reproductive event. The dynamics of the resources occur on a much faster time scale and these dynamics are followed in continu-

ous time within a year. Since the consumer density does not change on this time scale, resource densities reach a within-year equilibrium. First the dynamics of the consumer is introduced as a function of the, as yet unspecified, equilibrium densities of the resources. In a second step the resource dynamics and their equilibrium are derived.

The recurrence equation for the consumer is given by

$$
N_{t+1}=\left(\alpha_{1} C_{1}+\alpha_{2} C_{2}\right) N_{t},
$$

where $C_{i}$ is the functional response of the consumer for resource $i$. The constant $\alpha_{i}$ is the conversion efficiency of consumed resources into offspring. Prey consumption is modeled by applying a twospecies version of Holling's disk equation, resulting in a saturating (type II) functional response $C_{i}$ for each resource $i$ (Case 2000):

$$
C_{i}=\frac{e_{i} \hat{R}_{i t} p_{i} f_{i}}{1+e_{1} \hat{R}_{1 t} p_{1}\left(t_{\mathrm{p} 1}+f_{1} t_{\mathrm{m} 1}\right)+e_{2} \hat{R}_{2 t} p_{2}\left(t_{\mathrm{p} 2}+f_{2} t_{\mathrm{m} 2}\right)} \quad \text { for } \quad i \in\{1,2\} .
$$

The number of encountered prey per time step is the product of search efficiency $e_{i}$ and resource density $\hat{R}_{i t}$ in year $t$. Upon encounter the consumer decides to attack the prey with probability $p_{i}$. In Rueffler et al. (2006a) it was assumed that any prey is attacked upon encounter, i.e., $p_{1}=1=p_{2}$. In this paper the $p$-values are derived according to optimal diet choice theory and the detailed procedure is described in the next section. The capture probability $f_{i}$ represents the probability that an attacked prey is actually subdued. The handling time consists of two components: the pursuit time $t_{\mathrm{p} i}$ and the manipulation time $t_{\mathrm{m} i}$. The pursuit time is the time that is needed to get hold of a prey once it is detected. After the prey is caught it might still need a treatment before it can be consumed, the duration of the treatment is the manipulation time. The denominators of $C_{1}$ 
and $C_{2}$ are identical and can be factored out. This factor, to be referred to as search probability,

$$
s=1 /\left(1+e_{1} \hat{R}_{1 t} p_{1}\left(t_{\mathrm{p} 1}+f_{1} t_{\mathrm{m} 1}\right)+e_{2} \hat{R}_{2 t} p_{2}\left(t_{\mathrm{p} 2}+f_{2} t_{\mathrm{m} 2}\right)\right)
$$

is the fraction per time step spent searching for prey. Equation (1) can now be written as

$$
N_{t+1}=s\left(\alpha_{1} e_{1} p_{1} \hat{R}_{1 t} f_{1}+\alpha_{2} e_{2} p_{2} \hat{R}_{2 t} f_{2}\right) N_{t} .
$$

The resource dynamics within the foraging season in year $t$ is followed on a within-year time scale $\tau$ :

$$
\frac{\mathrm{d} R_{i t}}{\mathrm{~d} \tau}=b_{i}-d_{i} R_{i t}(\tau)-C_{i} N_{t} \quad \text { for } \quad i \in\{1,2\} .
$$

We assume that resource production is constant with influx $b_{i}$. The death rate is given by $d_{i}$. Since constant consumer densities $N_{t}$ are assumed within years, the following implicit description of the resource equilibria $\hat{R}_{i t}$ using equation (2) and (3) can be given:

$$
\hat{R}_{i t}=\frac{b_{i}}{d_{i}+e_{i} p_{i} f_{i} s N_{t}} \quad \text { for } \quad i \in\{1,2\} .
$$

In order to calculate the consumer and resource equilibria, one has to solve equation (4) and (5) simultaneously using equation (2) and (3). This can be done analytically but yields rather lengthy expressions that are not shown here (Mathematica notebook available on request).

\section{Diet choice}

A crucial parameter in this study is the probability of attack by the consumer upon encounter with an item of the $i$ th resource, $p_{i}$. This parameter has no counterpart in the models of Lawlor and Maynard Smith (1976), Lundberg and Stenseth (1985), Abrams (1986), and Wilson and Turelli (1986). These authors assume that consumers behave opportunistically. In Rueffler et al. (2006a) both $p$-values are explicitly set equal to one. In this paper adaptive consumer behavior is allowed: each individual chooses an optimal diet depending on its morphology and the abundance of the two resources. If consumers have perfect information and make optimal decisions, this procedure gives rise to the "zero-one rule", i.e., an individual either always or never attacks a certain resource (Stephens and Krebs 1986). How does this rule from the individual level extends to the population as a whole (Giraldeau and Caraco 2000)? In principle it is conceivable that a morphologically monomorphic population consists of a mixture of different feeding types, e.g. opportunists and selectors, at frequencies such that the fitness of all foraging strategies is equal. In appendix A we show that in the present model such a behavioral polymorphism cannot occur in a morphologically monomorphic population. At population dynamical equilibrium, either all individuals are selectors or all individuals are opportunists. In order to distinguish between these different cases, resources are assigned a profitability (Stephens and Krebs 1986). It is defined as the fitness gain a certain resource item entails for the consumer per investment of time:

$$
\rho_{i}=\frac{\alpha_{i} f_{i}}{t_{\mathrm{p} i}+f_{i} t_{\mathrm{m} i}}
$$


If pursuit time $t_{\mathrm{p} i}$ is negligible, profitability simplifies to $\rho_{i}=\alpha_{i} / t_{\mathrm{m} i}$. If both the pursuit and the handling time are negligible, attacking a prey is cost-free resulting in opportunistic consumers. For each individual consumer it is always beneficial to attack the resource with the higher profitability. The question is whether it also pays to attack the lower ranked resource as well. In appendix A it is shown that a population of specialists feeding on the more profitable resource $j$ will include the less profitable resource $i$ in its diet, when the latter could sustain a consumer population on its own (see also Vincent et al. (1996)) and that this condition is met when

$$
\rho_{i}>1
$$

How do consumers behave in a morphologically dimorphic population? This question becomes important when the emergence of rare mutants is considered. Due to its deviating morphology it might be beneficial for a mutant to also deviate in its behavior. This possibility will influence both a mutant's probability of invasion (see next section) and its scope to coexist with the resident. Here it is assumed that individuals are able to adjust their behavior instantaneously and that it takes no learning period to get perfect knowledge on the environmental condition. Whether a mutant only attacks the resource with the higher profitability or also feeds on the lower ranked resource, crucially depends on the resource abundances it encounters and therefore on the morphological traits and the behavior of the resident (appendix A). Once a morphologically dimorphic population has reached its ecological equilibrium, the behavior of each type is again determined by condition (8).

\section{Evolutionary dynamics}

As in Rueffler et al. (2006a), the evolutionary dynamics of five different foraging-related traits are studied separately (see table 2). Each trait consists of two resource-specific components. For instance, capture probability can be measured with respect to resource 1 and resource 2 , denoted $f_{1}$ and $f_{2}$. Often we will write such a pair of traits as a vector: $\boldsymbol{f}=\left(f_{1}, f_{2}\right)$. We assume that evolution proceeds along a trade-off curve that constrains covariation in the two traits (fig. 1). An increase in one resource-specific component is accompanied by a decrease in the other. The trait combinations $\boldsymbol{x}=\left(x_{1}, x_{2}\right)$, where $\boldsymbol{x} \in\left\{\boldsymbol{e}, \boldsymbol{f}, \boldsymbol{t}_{\mathrm{p}}, \boldsymbol{t}_{\mathrm{m}}, \boldsymbol{\alpha}\right\}$, on this curve in the $\left(x_{1}, x_{2}\right)$-space are parameterized by a specialization coefficient $\theta$ that varies continuously between 0 and 1 . For given resource densities, specialization for resource $i$ corresponds to an increase in the functional response $\alpha_{i} C_{i}$, that is, to an increase in either $\alpha_{i}, e_{i}$ or $f_{i}$ or to a decrease in $t_{\mathrm{m} i}$ or $t_{\mathrm{p} i}$ (see eq. 1 ). Therefore we have to use different parameterizations. If $\boldsymbol{x} \in\{\boldsymbol{\alpha}, \boldsymbol{e}, \boldsymbol{f}\}$, then $\boldsymbol{x}=\left(x_{1 \max }(1-\theta)^{\frac{1}{z}}, x_{2 \max } \theta^{\frac{1}{z}}\right)$ and if $\boldsymbol{x} \in\left\{\boldsymbol{t}_{\mathbf{p}}, \boldsymbol{t}_{\mathbf{m}}\right\}$, then $\boldsymbol{x}(\theta)=\left(x_{1 \max }-x_{1 \min }(1-\theta)^{\frac{1}{z}}, x_{2 \max }-x_{2 \min } \theta^{\frac{1}{z}}\right)$, where $x_{1 \min }, x_{1 \max }, x_{1 \min }, x_{2 \min }$ are positive constants. These parameterizations are such that $\theta=0$ corresponds to a specialist for resource 1 while $\theta=1$ corresponds to a specialist for resource 2. The positive parameter $z$ determines the curvature of the trade-off curve. The trade-off curve is linear when $z=1$. Furthermore, $z>1$ corresponds to a weak trade-off, while $z<1$ corresponds to a strong trade-off (fig. 1). In comparison 
with a linear trade-off curve, generalists with $\theta=0.5$ have lower (higher) total functional response in case of a strong (weak) trade-off. Throughout this article both the vector $\boldsymbol{x}=\left(x_{1}, x_{2}\right)$ and the specialization coefficient $\theta$ are used to characterize a pair of resource specific traits on a trade-off curve.

The possibility that a rare mutant invades a resident community depends on its invasion fitness $w$, i.e., its long term per capita growth rate in an environment where the resource densities are determined by the trait values and the behavior of the resident consumers (e.g. Metz et al. 1992). The growth rate of a mutant depends on its foraging morphology and its behavior. It might therefore be tempting to account explicitly for the effect of both morphology and behavior in the notation of the fitness function by writing $w\left(\theta^{\prime}, \boldsymbol{p}^{\prime}, \theta, \boldsymbol{p}\right)$. However, this notation would be misleading because it suggests that $\boldsymbol{p}$ is a free parameter that can vary independently of $\theta$. This is not the case for two reasons. Firstly, from inequality (8) we can conclude that the diet composition of a resident consumer population is fully determined by its morphology. In other words, at population dynamical equilibrium of consumers and resources diet choice is an emergent property of morphology. This implies that also the resource equilibria $\hat{R}_{i}$ (eq. 6) are fully determined by the $\theta$-value of the resident consumers. Secondly, from equation (A1) in appendix A follows that the diet choice of mutants is determined by their morphology and by the resource densities as set by the resident consumers. Hence, invasion fitness is fully determined by the specialization coefficients of the mutant and the resident. For $\boldsymbol{x} \in\left\{\boldsymbol{e}, \boldsymbol{f}, \boldsymbol{t}_{\mathrm{p}}, \boldsymbol{t}_{\mathrm{m}}\right\}$ invasion fitness is given by

$$
w\left(\theta^{\prime}, \theta\right)=\alpha_{1} C_{1}\left(\theta^{\prime}, \hat{R}_{1}(\theta), \hat{R}_{2}(\theta)\right)+\alpha_{2} C_{2}\left(\theta^{\prime}, \hat{R}_{1}(\theta), \hat{R}_{2}(\theta)\right),
$$

where $C_{i}$ is given by equation $(2)$ and $\hat{R}_{i}(\theta)$ denotes resource equilibria across years set by a consumer with trait value $\theta$. For $\boldsymbol{x}=\boldsymbol{\alpha}$ the $\alpha_{i}$ 's are functions of $\theta^{\prime}$ instead of the $C_{i}$ 's.

We assume that mutations are rare and of small effect. Because of the first assumption the ecological and evolutionary time scales are separated: a population has reached its ecological equilibrium before a new mutant arises. This approximation is an essential prerequisite for using the toolbox of adaptive dynamics (Metz et al. 1996; Geritz et al. 1998; Waxman and Gavrilets 2005). Both assumptions together assure that the evolutionary dynamics of clonal populations consisting of a single phenotype, is equivalent to those of a diploid sexual model with additive genetics (Van Dooren in press; Metz in press).

Mutants with $w\left(\theta^{\prime}, \theta\right)>1$ can invade while mutants with $w\left(\theta^{\prime}, \theta\right)<1$ certainly go extinct. The direction of evolutionary change can be derived from the fitness gradient, i.e., the first derivative of the fitness function (eq. 9) with respect to the mutant's trait evaluated at the resident's trait. Trait values $\theta^{*}$ where the fitness gradient equals zero, that is,

$$
0=\left.\frac{\partial w\left(\theta^{\prime}, \theta^{*}\right)}{\partial \theta^{\prime}}\right|_{\theta^{\prime}=\theta^{*}}
$$

are of special interest. These are called evolutionarily singular points by Metz et al. (1996) and Geritz et al. (1998) and they can be classified according to two independent properties: convergence 
stability and invadability (Geritz et al. 1998; Rueffler et al. 2004). The first property determines whether a singular trait value is approached from nearby (Eshel 1983; Christiansen 1991; Abrams et al. 1993; Geritz et al. 1998) while the second property determines whether any consumer with a different trait value can increase in frequency when initially rare in a population dominated by individuals with $\theta^{*}$ (Maynard Smith 1982). Singular trait values that are both convergence stable and uninvadable are final stops of evolution and were named continuously stable strategies or CSSs by Eshel and Motro (Eshel and Motro 1981; Eshel 1983) while singular points that lack both properties are evolutionary repellors. A convergence stable and invadable trait value is called an evolutionary branching point (Metz et al. 1996; Geritz et al. 1998). At these points selection becomes disruptive and favors increased genetic variation (Rueffler et al. 2006b).

\section{Results}

From equation (7) we can see that the profitabilities $\rho_{i}$ of the resources depend on $\alpha_{i}, f_{i}, t_{p \mathrm{i}}$ and $t_{m \mathrm{i}}$ but not on the search efficiencies $e_{i}$. Profitabilities are independent of search efficiencies because of the assumption made in optimal foraging theory that encounter without attack takes no time and entails no extra cost (Stephens and Krebs 1986). Therefore the frequency of encounter has no influence on the decision to attack a prey. Depending on the values of the other traits, either both resources or only one of them is attacked. If both resources are attacked, evolutionary dynamics are identical to those reported in Rueffler et al. (2006a), where diet choice is always opportunistic. If only one resource is attacked, then the consumer population experiences directional selection towards specialization in search efficiency for the chosen prey, independent of the initial condition and the curvature of the trade-off.

For the four other traits, behavior does depend on the trait values of the consumer and can therefore change as traits evolve. For the remainder of this paper we make the assumption that all non-evolving traits and fixed parameters are symmetric with respect to the two resources. Then consumers characterized by $\theta=0.5$ equalize the profitabilities $\left(\rho_{1}=\rho_{2}\right)$ and we refer to them as generalists. Asymmetric parameter values do not change the results qualitatively but merely make figures 2, 3, 4, 6 and 7 less symmetric. Generalists attack both types of prey upon encounter. The question is whether consumers to whom one resource is more profitable than the other will include the less profitable resource in their diet. From equation (7) we see that the two profitabilities are monotone functions of the trait values and therefore also of the specialization coefficient $\theta$. This means that with increasing specialization the profitability of one resource continuously increases while the profitability for the other continuously decreases. The less profitable resource is dropped from the diet as soon as its profitability decreases below 1 (eq. 8). It follows that consumers behave as an opportunist in an interval around $\theta=0.5$, as a selector for resource $1(\boldsymbol{p}=(1,0))$ for values of $\theta$ sufficiently close to 0 and as a selector for resource $2(\boldsymbol{p}=(0,1))$ for values of $\theta$ sufficiently close to 1 . From figure 2 we can see that the region of opportunistic behavior grows with decreasing strength of the trade-off (with increasing values of $z$ ). Because weak trade-offs 
correspond to high profitabilities $\rho_{i}\left(e_{i}, f_{i}\right.$ and $\alpha_{i}$ are increasing functions of $z$, while $t_{\mathrm{p} i}$ and $t_{\mathrm{m} i}$ are decreasing functions of $z$, see figure 1), they make it more likely that the condition for opportunistic behavior (eq. 8) is met.

Resident consumers switch from opportunistic to selective behavior when inequality (8) turns into an equality. For such a $\theta$-value the two behavioral alternatives yield exactly the same fitness and the probability to attack the less suitable resource can take any value between zero and one without affecting fitness. At these switch points, not only the behavior of residents changes discontinuously but also that of mutants (fig. 3). Therefore the fitness gradient (eq. 10) is not defined. These trait values are named "degenerate singular points" here, because they are critical points of the fitness function but lack differentiability. The model behavior is special at these degenerate singular points, as described below.

We first discuss the consequences of flexible diet choice behavior for the evolutionary dynamics of monomorphic populations. In the central region of the trait space where consumers behave opportunistically the model is identical to our previous model where diet choice was absent (Rueffler et al. 2006a). Hence, the generalist with $\theta=0.5$ is a CSS for weak trade-offs. In case of strong tradeoffs the generalist is a branching point of the evolutionary dynamics for capture probability $f$ and an evolutionary repellor for conversion efficiency $\boldsymbol{\alpha}$, pursuit time $\boldsymbol{t}_{\mathrm{p}}$ and manipulation time $\boldsymbol{t}_{\mathrm{m}}$ (fig. $2 \& 3$ ). In the boundary region of the trait space where consumers behave selectively they do not feel any trade-off. In this situation selection favors further specialization in all traits and independently of the curvature of the trade-off (fig. $2 \& 3$ ). Hence, any ancestral population of selectively behaving individuals evolves towards complete morphological specialization and convergence stable singular points (CSSs and branching points) are not globally attracting anymore but only attract within the interval where the resident consumer behaves opportunistically. As a consequence, the possibility that a polymorphism emerges via an evolutionary branching point depends on initial conditions (fig. $2 \mathrm{a} \& 3 \mathrm{~b}$ ).

We now turn to the evolutionary dynamics of dimorphic populations. A major result of Rueffler et al. (2006a) was that coexistence of different phenotypes is possible when these differ sufficiently in either search efficiency $\boldsymbol{e}$ or capture probability $\boldsymbol{f}$ while phenotypes that only differ in conversion efficiency $\boldsymbol{\alpha}$, pursuit time $\boldsymbol{t}_{\mathrm{p}}$ and manipulation time $\boldsymbol{t}_{\mathrm{m}}$ cannot coexist. In the first case coexistence is mediated by negative frequency-dependent interactions. Frequency dependence is not present in populations dimorphic for $\boldsymbol{\alpha}, \boldsymbol{t}_{\mathrm{p}}$ and $\boldsymbol{t}_{\mathrm{m}}$. In appendix B we prove that negative frequency dependence is present for any trait whenever the morphologies are sufficiently different such that genotypes differ in their diet choice. Hence, flexible diet choice can mediate coexistence of different phenotypes that could not coexist in the absence of such behavior.

Whether the behavior of a rare mutant type deviates from that of the resident is indicated in the PIPs of figure 3 by mutant choice boundaries. These lines are defined by an equality in the switching conditions (eq. [A1] in appendix A). The two types in a given mutant-resident combination differ in their behavior when a vertical line drawn from the main diagonal at the resident's trait value to the 
mutant's trait value crosses a mutant choice boundary. After the successful invasion of a mutant two scenarios have to be distinguished. The individuals of a successful mutant population either stick to their new behavior until the new population dynamical attractor is reached or they switch behavior again once their population size has passed a certain threshold. Which scenario applies to a given mutant-resident combination can also be deduced from the mutant choice boundaries. The first happens when the mutant is sufficiently specialized such that it would behave selectively if it were the only resident (fig. $3 \& 4$ ). The latter happens when the mutant trait lies in the region of trait space where trait values correspond to a selector when they are rare and to an opportunist when they are the resident (fig. $3 \& 4$ ). In this case a horizontal line drawn from the mutant's trait to the main diagonal crosses a mutant choice boundary again.

Once a population is dimorphic, the further co-evolutionary dynamics of the two types can be read from figure 4. The depicted plots show the sets of pairs of phenotypes that are able to coexist in a protected dimorphism. These sets are given by the overlapping parts of the ' + ' region of a pairwise invadability plot (PIP) and its mirror image taken along the main diagonal (for further details see Metz et al. (1996); Geritz et al. (1998, 1999)). Note that the plots in figure 4 are symmetric with respect to the main diagonal. The two symmetric parts show the same dynamics but with a reversed numbering of the two types. The direction of selection acting on two coexisting types, indicated by the arrows in figure 4, can be derived graphically with a geometrical method described in Rueffler et al. (2004), but taking into account whether a mutant changes behavior or not.

Polymorphisms can emerge in two different ways. Firstly, in case of capture probability $f$ a dimorphism can emerge at an evolutionary branching point (figs. $2 \& 3$ ). In the initial phase after branching the coexisting types remain opportunists (figs. 4a \& 5a). In the course of further divergence both types become genetically more specialized and each type approaches the parameter region where prey selection pays off. When the rate of evolution differs in the two types, one of them turns into a selector while the other is still an opportunist. This results in a population with "nested" behavior where the diet of the selector consists of a subset of the diet of the opportunist. Both types still experience directional selection towards further specialization and finally the population will reach a continuously stable coalition with two extreme specialists that are selectors for different resources (fig. 4a). To check whether these results are robust against deviations from the assumption of strict mutation limitation, we present some individual-based simulations in figure 5 (see appendix $\mathrm{C}$ for details). In figure 5a the simulation starts with an opportunistically behaving monomorphic population characterized by $\theta=0.35$. Initially evolutionary change occurs in the direction of the generalist. Then phenotypes on opposite sides of the branching point at $\theta=0.5$ can establish, giving rise to two diverging lineages. The lineage specializing on resource 2 turns into a selector slightly earlier than the other lineage specializing on resource 1 does. Once both types behave selectively, rapid evolution leads to complete specialization.

Secondly, polymorphisms can emerge when a mutant appears that differs sufficiently from the resident in its morphology and, as a consequence, starts to behave differently. To see this we have 
to compare figures 3 and 4 . For most mutant-resident combinations where the mutant is able to invade and where it differs in its behavior from the resident (fig. 3) the two types are able to coexist in a protected polymorphism (fig. 4). In case of strong trade-offs this route to polymorphism also leads to a coalition of two selectors that are each completely specialized on a single resource (fig. $4 \mathrm{a}, \mathrm{c})$. With weak trade-offs an alternative evolutionarily stable coalition exists due to the presence of evolutionary attracting isoclines (fig. 4b,d). Isoclines are found at the zeroes of the dimorphic fitness gradient (Geritz et al. 1998, 1999):

$$
0=\left.\frac{\partial w\left(\theta^{\prime}, \theta^{1}, \theta^{2}\right)}{\partial \theta^{\prime}}\right|_{\theta^{\prime}=\theta^{i}} .
$$

Superscripts 1 and 2 refer to the two coexisting types of a dimorphic resident community, and isoclines for changes in resident $1(i=1)$ and resident $2(i=2)$ can exist. However, in our case a single isocline exists for the opportunists in the region of the phase plane where one type behaves as an opportunist while the other behaves as a selector (fig. 4b,d). In dimorphic populations residing on such an isocline the opportunist cannot be invaded by any other type while the selector experiences directional selection just as elsewhere. Whenever the selectors become invaded by a more specialized type the trait values of the residents no longer reside on the isocline and opportunists become susceptible to invasion by mutants that improve on the resource neglected by the selector. Isoclines attach to the boundary of the set of phenotypes that can coexist vertically above and below and horizontally to the left and to the right of the singular point at $\theta=0.5$ (fig. 4b,d) (Geritz et al. 1999). If the trade-off is sufficiently weak, the isoclines extend to the boundary of the trait space $(z=1.5$, fig. $4 \mathrm{~d})$. This boundary point constitutes an alternative evolutionarily stable coalition where one type is a specialized selector and the other type behaves opportunistically and is morphologically intermediate with a bias towards the resource not taken by the selector. With decreasing values of $z$ the opportunist in the coalition becomes increasingly specialized on the resource not taken by the selector until it finally pays for the opportunist to become a selector as well $(z=1.2$, fig. $4 \mathrm{~b})$. At this point the coevolutionary dynamics enters the region of the trait space where both types behave selectively, resulting in complete morphological specialization. In figure $5 \mathrm{c}$ we demonstrate this outcome with individual-based simulations. Since in the case of weak trade-offs a polymorphism cannot emerge via mutations of small effect, the simulation is initialized by adding 10 immigrants characterized by $\theta=0.15$ to a resident consumer population at its ecological equilibrium of 9100 individuals characterized by $\theta=0.25$.

Although no branching point in the sense of the adaptive dynamics literature (Metz et al. 1996; Geritz et al. 1998) exists for $\boldsymbol{\alpha}, \boldsymbol{t}_{\mathrm{p}}$ and $\boldsymbol{t}_{\mathrm{m}}$, a polymorphism can nevertheless evolve through a series of mutations of small effect. To see this, consider a scenario with an ancestral type characterized by a value of $\theta$ slightly smaller than 0.5 . This phenotype behaves opportunistically and selection favors mutants with smaller $\theta$-values, i.e., types with a shorter manipulation time for resource 1 (see fig. $2 \mathrm{~b} \& 3 \mathrm{c}$ ). At some point evolution has driven the population so close to the degenerate singular point that a mutant with a smaller trait value than the resident drops resource 2 from its diet (fig. 3c). Because resident and mutant differ in their behavior they can coexist despite of their 
small morphological difference (fig. 4c). Now character displacement between the two phenotypes takes place and the selector becomes completely specialized for resource 1 while the opportunist evolves in the opposite direction to eventually become a specialist for resource 2. In this scenario degenerate singular points give rise to dimorphisms through a series of mutations of small effect, a property that was known previously only from evolutionary branching points. This scenario is confirmed by individual-based simulations (fig. 5b).

\section{Adding foraging inaccuracy}

Empirical tests of optimal diet choice theory revealed that prey choice is better described by sigmoid functions than by the step functions reflecting the zero-one rule (Krebs et al. 1977). Deviations from the theoretical predictions of optimal foraging theory can be expected for several reasons. Consumers are likely to have incomplete information about the abundance of resources and imprecise estimates about their profitabilities, i.e., about their own performance with respect to the different resources. Here, we investigate the robustness of our results with respect to deviations from the zero-one rule by assuming that consumers do have perfect information but make mistakes in their decisions and that the magnitude of these mistakes increases with decreasing cost of non-optimal behavior, as determined by the fitness difference between opportunistic and selective behavior. We implement this semi-mechanistic argument by using the following equation to describe the probability of attack:

$$
p_{i}=\left[1+\exp \left(a\left(\frac{\alpha_{j} e_{j} \hat{R}_{j}(\theta) f_{j}}{1+e_{j} \hat{R}_{j}(\theta)\left(t_{\mathrm{p} j}+f_{j} t_{\mathrm{m} j}\right)}-\rho_{i}\right)\right)\right]^{-1} .
$$

When $p_{i}$ is plotted as function of the specialization coefficient $\theta$ we get a sigmoid curve with $p_{i}=0.5$ at the trait value where an optimally behaving consumer would switch in its diet choice (fig. 6, first column). The foraging accuracy parameter $a$ determines to what extent diet choice deviates from the zero-one rule. If $a \rightarrow \infty$, perfect decision-making is approached and if $a \rightarrow 0$, prey choice becomes increasingly random. With the introduction of sigmoid prey switch curves the mutant choice boundaries in figure 3 and the dashed lines in figure 4, which both reflect the abrupt switch in prey choice of accurately behaving individuals, no longer make sense. The fitness function becomes differentiable at these points and the kinks in the contour lines of the fitness function get rounded (fig. 6). When the accuracy parameter $a$ decreases diet choice becomes more and more balanced and therefore the evolutionary dynamics becomes more and more similar to that found in the absence of diet choice (Rueffler et al. 2006a). In cases with a convergence stable generalist (CSS or a branching point) the basin of attraction of the generalist grows with decreasing accuracy. This entails shrinking basins of attraction for the two specialists (fig. 6). In case of a repelling generalist, that is, for strong trade-offs in combination with evolving manipulation time $\boldsymbol{t}_{\mathrm{m}}$, pursuit time $\boldsymbol{t}_{\mathrm{p}}$ or conversion efficiency $\boldsymbol{\alpha}$, the situation is more complicated. Two qualitatively different ways exist in which a degenerate singular point can unfold. Figure $7 \mathrm{~b}$ shows the first scenario 
in which the degenerate singular points disappear. The generalist strategy becomes immediately globally repelling, as in the case without prey choice. Figure 7c shows the second scenario in which a slight inaccuracy changes the degenerate singular points into a CSS and a repellor.

Overall the co-evolutionary dynamics of two coexisting types does not change qualitatively when foraging inaccuracy is introduced. In all cases the combination of two highly specialized selectors remains a continuously stable coalition. Also the location of the evolutionary isocline that occurs in combination with weak trade-offs does not change qualitatively. Hence, the alternative coalition consisting of one opportunist and one selector remains convergence stable when tradeoffs are sufficiently weak. Only the emergence of polymorphisms by small mutational steps at degenerate singular points as described in the previous section is hampered. That scenario relied on the fact that a mutant that only slightly differs in its morphology from the resident can differ in its behavior from the resident. When foraging inaccuracy causes the degenerate singular point to disappear altogether (fig. 7b), mutants have to differ more strongly from the resident in order to enter the area of coexistence compared to the case without foraging inaccuracy (fig. 7c). When foraging inaccuracy causes the degenerate singular point to give rise to a CSS and a repellor (fig. 7d), a small mutational step can still lead into the area of coexistence (fig. 7e). However, in this case two new evolutionary isoclines appear that not only prevent further divergence of the newly established type but even impose convergent selection such that the dimorphic population eventually collapses to become monomorphic again (fig. 7f). Only mutants that lie beyond these isoclines can give rise to dimorphisms that undergo disruptive selection.

\section{Discussion}

This paper focuses on the consequences of behavioral diet choice for the evolution of various foraging traits in a consumer facing two different resources. A mathematical model is analyzed that builds on an existing model for the evolution of morphological characters (Rueffler et al. 2006a). In the original model consumers behave opportunistically and every resource item is attacked upon encounter. In this study each individual chooses to attack resource items so as to maximize its energy intake.

The results show that the added feature of behavioral flexibility affects various aspects of the evolutionary dynamics. (i) Behavior guides the direction of selection. Only resources that are included in the diet entail a selective force. Consumers that choose few resources will also become specialized in terms of their evolving traits to exploit these resources efficiently. This aspect has been noted earlier (e.g. Stenseth 1984; Abrams 1986; Brown 1990). (ii) A convergence stable singular point (CSS or branching point), will only be approached when the ancestral population behaves opportunistically. Whenever the ancestral population behaves selectively, directional selection will lead towards specialized morphologies. Therefore diet choice reduces the likelihood that a population evolves to become a generalist and whether a population can diversify via a branching point depends on initial conditions. (iii) Successful mutants that differ in their behavior from the 
resident will in most cases not replace the resident but coexist with it in a protected polymorphism. The emergence of polymorphisms mediated by differential diet choice can occur for a wide range of parameters through the immigration of phenotypes that deviate sufficiently from the resident or through mutations of large effect. However, this mechanism can also create polymorphism in the absence of immigration with mutational steps constrained to be small. This happens whenever a population evolves towards a degenerate singular point where the less profitable resource becomes so unattractive that a slightly more specialized mutant turns into a selector. This process, which generically gives rise to to polymorphisms through a series of mutations of small effect, has to our knowledge not been described previously. (iv) The incorporation of flexible diet choice enlarges the set of conditions allowing for coexistence. Whenever two types have mutually exclusive diets, coexistence is guaranteed. Furthermore, coexistence is possible when the two types have nested diets, i.e., when one type feeds on both resources while the other selects only one of them. For conversion efficiency $\boldsymbol{\alpha}$, pursuit time $\boldsymbol{t}_{\mathrm{p}}$ and manipulation time $\boldsymbol{t}_{\mathrm{m}}$ diet choice is the sole mechanism to mediate coexistence. (v) In models lacking diet choice the only continuously stable coalition (i.e., attracting and co-evolutionarily stable community) consists of two extreme specialists (Lawlor and Maynard Smith 1976; Abrams 1986; Rueffler et al. 2006a). In the present model two additional continuously stable coalitions exist given sufficiently weak trade-offs. These consist of one selecting specialist and one opportunistic intermediate consumer, which is biased in its morphology towards the resource that is neglected by the specialist.

Some related models have been analyzed previously but with different emphasis. To our knowledge, Stenseth (1984) was the first to combine the evolutionary dynamics of consumer traits with optimal diet choice theory. Based on qualitative arguments he concluded that abundant resources that are difficult to handle favor selective specialists while easy to handle and scarce resources select for opportunistic generalists in case of weak trade-offs and for opportunistic specialists in case of strong trade-offs. Stenseth's model does not incorporate explicit resource dynamics and therefore does not allow for dynamic feedbacks between consumer and resource densities and diet choice behavior. Brown $(1990,1996)$ considered a consumer in a patchy environment that can specialize in terms of its morphology on different patch types and that can choose which patch type to exploit. He investigates the possible monomorphic and polymorphic evolutionary endpoints that such a system can adopt. Brown's model is special in so far as it allows for a mixture of opportunists and selectors in a morphological monomorphic population, an impossible constellation in the model analyzed here. At such trait values the population is prone to become morphologically polymorphic because selectors and opportunists experience very different selection pressures. Vincent et al. (1996) investigated the scope for coexistence of different consumer types in a model that yields a fitness function equivalent to the one analyzed here. Although these authors allow for behavioral diet choice, they dismiss the possibility that this mechanism mediates coexistence. In the model version where Vincent et al. consider mixed substitutable resources, they limit their analysis to the region of the trait space where consumers behave opportunistically. They argue that resources that are not included in the diet cannot be considered a resource because they cannot sustain a consumer population on their own. Our results show that this viewpoint excludes a set of inter- 
esting results when populations evolve such that resources become excluded from or included in the diet over evolutionary time. More recently, Svanbäck and Bolnick (2005) analyzed a simple population genetic model for the evolution of handling time. These authors show that diet choice can lead to disruptive selection across a wide range of conditions. However, Svanbäck and Bolnick keep consumer dynamics constant and therefore cannot explore the consequences of the feedback between population dynamics, diet choice, and trait evolution. Abrams and coworkers (Abrams 1999; Abrams and Matsuda 2003, 2004) focus on population dynamical systems of one or more consumer types feeding on two prey where the population dynamics do not reach a stable equilibrium. They show that non-instantaneous diet choice or relatively fast evolutionary changes can cause complex population dynamics and thereby lead to unexpected mean trait values. Abrams (2006) shows that under these conditions even trimorphisms can readily occur.

Optimal foraging theory predicts that resources with a low profitability are neglected by consumers when resources with a higher profitability are sufficiently abundant (Stephens and Krebs 1986). This prediction is valid at the ecological time scale where resource densities vary in time. The prediction of optimal foraging theory does not apply when consumer and resource populations regulate each other such that they reach stable population dynamical equilibria. In this situation the resource densities are not free parameters but functions of the evolving consumer traits. This explains why a resource is included in the diet by a resident consumer when the profitability $\rho_{i}$ is greater than unity (eq. 8), a condition that purely depends on consumer traits and not on the population dynamics of the resource (eq. 7). Therefore, if we account for the feedback between consumer and resource densities, the statement of Stenseth (1984) that abundant resources favor selective consumers that subsequently undergo morphological specialization is not relevant. In the model presented here, the ecological time scale does apply during the process of invasion. An initially rare mutant type does not affect the resource densities yet. In this phase it can be beneficial to feed selectively on the more profitable resource. With increasing frequency of the mutant type, it depletes its preferred resource so that in the end, when it reaches fixation, it may become beneficial to also forage on the less suitable resource.

Our model is based on the assumption of clonal genetics. In the limit of rare mutations with small phenotypic effect and random mating, the results apply to monomorphic diploid sexual populations and polygenic traits. In case of polymorphic sexually reproducing populations, the course of evolution cannot be predicted straightforwardly from a clonal model. However, some predictions can still be made. Intermediate heterozygotes will behave as either opportunists or selectors. Whenever such heterozygotes are inferior, disruptive selection favors any mechanism that prevents the production of heterozygotes (Rueffler et al. 2006b). Processes such as the evolution of assortative mating or dominance modification lead to two discrete phenotypes and in this situation we expect that long term evolution will lead to the evolutionary endpoints predicted by the clonal model. The condition of heterozygote inferiority in a behaviorally polymorphic population is also given in a model by Svanbäck and Bolnick (2005) where it indeed imposes disruptive selection. The problem of heterozygote inferiority is circumvented altogether when disruptive selection leads 
to a phenotypic dimorphism that does not require a new genetic polymorphism (Rueffler et al. $2006 b$ ). In, for example, a sexual dimorphism, a polymorphism emerges from a population with undifferentiated sexes by a mutation in sex-specific genes which are already present. If the two sexes become sufficiently different in terms of their morphology they will also chose different resources and subsequently undergo further morphological differentiation (Bolnick and Doebeli 2003; Van Dooren et al. 2004).

The presented model is based on three different limits. (i) Mutations are rare. This allows for the separation of ecological and evolutionary time scale. (ii) Foraging accuracy is high. This leads to the zero-one rule of diet choice. (iii) Learning is fast so that the optimal behavior is adopted immediately. Of course none of these limits is a proper description of reality. They are primarily considered because they make the model mathematically tractable. Investigating such limits is generally informative as a guideline for understanding the phenomena we observe in nature but it is important to explore how violation of the limiting situation alters the model behavior. We show that our results are robust against deviations from the first limit by means of individualbased simulations. In these simulations evolution is not strictly mutation limited and new mutants frequently arise before previous ones have gone to fixation. These simulations also account for the effects of drift and of stochastic changes in population size. Deviations from the second limit are investigated by introducing inaccurately foraging consumers. In this case the step-like prey switch reflecting the zero-one rule is replaced by a sigmoidal switch and we show that moderate inaccuracies do not change the qualitative model results. The third limit is the one most difficult to explore because it corresponds to a change in behavior within an organisms life span. If individuals need a long learning period before they behave optimally, then the extinction probability of rare mutants might increase. This would slow down the evolutionary dynamics.

In all scenarios considered here, traits evolved one at a time and not simultaneously as in natural systems. The simultaneous evolution of several traits might lead to different predictions. Imagine a scenario where a trait that allows for evolutionary branching, e.g. search efficiency $\boldsymbol{e}$, jointly evolves with a trait that does not allow for evolutionary branching, e.g. manipulation time $\boldsymbol{t}_{\mathrm{m}}$. Assume further that no correlation exists between $\boldsymbol{e}$ and $\boldsymbol{t}_{\mathrm{m}}$ and that therefore the trait space can be parameterized in two independent specialization coefficients, one describing the degree of specialization in terms of $\boldsymbol{e}$ and one in terms of $\boldsymbol{t}_{\mathrm{m}}$. If both trade-offs are strong, then selection in $\boldsymbol{e}$ acts in the direction of the generalist's trait where a population would subsequently experience disruptive selection, while selection in $\boldsymbol{t}_{\mathrm{m}}$ acts towards increasing specialization. Under these assumptions the phenotype that corresponds to a generalist in both $\boldsymbol{e}$ and $\boldsymbol{t}_{\mathrm{m}}$ turns into a saddle point of the two-dimensional evolutionary dynamics. In this case polymorphisms can arise in two different ways. If evolution in search efficiency proceeds faster than in manipulation time, then the population will evolve towards the branching point and become dimorphic in search efficiency. On the contrary, if evolutionary change occurs faster for manipulation time than for search efficiency, then the population will evolve towards a degenerate singular point and the population will become dimorphic in manipulation time as soon as a mutant appears that behaves 
as selector. In either case, whenever the population is dimorphic in one trait, selection favors a corresponding diversification in the other trait. Eventually the population will consist of two types, a selector for resource 1 with high search efficiency and a low manipulation time for this resource and a selector for resource 2 with the corresponding adaptations in search efficiency and manipulation time. In this example, the evolutionary endpoint does not differ from that predicted based on the evolution of either trait in isolation. However, the adopted route to this endpoint appears to be sensitive to initial conditions and to details of the mutational process.

To summarize, this paper shows that behavioral flexibility in diet choice can influence various aspects of the evolutionary dynamics of morphological consumer traits. Behavior guides natural selection since only resources that are preyed upon contribute to the direction of selection a consumer experiences. Furthermore, it is shown that diet choice behavior can mediate coexistence of different consumer types that could not coexist when diet choice is fixed. The effect of flexible diet choice on the emergence of polymorphism is twofold. On the one hand, flexible diet choice decreases the basin of attraction of evolutionary branching points. On the other hand, successful mutants that differ in their diet choice from residents are able to coexist in a protected polymorphism. This mechanism can even generate polymorphisms through a series of mutations of small effect. The dynamics of co-evolving consumer populations can lead not only to a community of two selectively behaving specialists but also to a community of a selective specialist and a more intermediate opportunistic phenotype.

\section{Acknowledgments}

CR was supported by the Research Council for Earth and Life Sciences (ALW), which is subsidized by the Netherlands Organization for Scientific Research (NWO) and by a Discovery Grant to P. Abrams from the Natural Sciences and Engineering Research Council of Canada. TVD was supported by a Dutch NWO-VENI grant. CR thanks Michel Durinx and Frans Jacobs for helpful discussions and Peter Abrams for comments.

\section{Appendix A: Diet Choice}

Each consumer individual always attacks the resource with the higher profitability $\rho_{i}$ (eq. 7). Consider the case $\rho_{1}>\rho_{2}$. A population of selectors for resource 1 can be invaded by opportunistically behaving individuals with the same morphology if

$$
\rho_{2}>\frac{\alpha_{1} e_{1} \hat{R}_{1}(\theta,(1,0)) f_{1}}{1+e_{1} \hat{R}_{1}(\theta,(1,0))\left(t_{\mathrm{p} 1}+f_{1} t_{\mathrm{m} 1}\right)},
$$

where $\hat{R}_{i}(\theta,(1,0))$ indicates the equilibrium density of the $i$ th resource as it results from consumption by a monomorphic consumer population with trait-value $\theta$ and behavior $\boldsymbol{p}=(1,0)$ at its 
equilibrium $\hat{N}$. The right-hand side of inequality (A1) per definition equals one because it describes the the per capita growth rate of a consumer at ecological equilibrium. Hence, condition (A1) simplifies to $\rho_{2}>1$ (eq. 8). The profitability $\rho_{i}$ is the limit of the per capita growth rate of a selector for resource $i$ for large resource abundance:

$$
\lim _{R_{i} \rightarrow \infty} \frac{\alpha_{i} e_{i} R_{i} f_{i}}{1+e_{i} R_{i}\left(t_{\mathrm{p} i}+f_{i} t_{\mathrm{m} i}\right)}=\frac{\alpha_{i} f_{i}}{t_{\mathrm{p} i}+f_{i} t_{\mathrm{m} i}}
$$

Therefore, inequality (A1) is equivalent to demanding that resource two can sustain a viable population on its own given the resource is sufficiently abundant. In case condition (A1) is fulfilled, one has to check whether opportunists will replace selectors or whether both behaviors can coexist, i.e., whether the two behavioral strategies can invade each other. Mutual invadability is given when next to condition (A1) also the following condition holds:

$$
\rho_{2}<\frac{\alpha_{1} e_{1} \hat{R}_{1}(\theta,(1,1)) f_{1}}{1+e_{1} \hat{R}_{1}(\theta,(1,1))\left(t_{\mathrm{p} 1}+f_{1} t_{\mathrm{m} 1}\right)} .
$$

For this model we prove that in a morphologically monomorphic population at population dynamical equilibrium all individuals behave either as selectors or as opportunists, that is, the conditions (A1) and (A2) cannot be fulfilled simultaneously. This follows from the fact that if $\rho_{1}>\rho_{2}>1$, then the density of resource 1 in the presence of an opportunistic consumer population, $\hat{R}_{1}(\theta,(1,1))$, is lower than in the presence of a population that feeds selectively on resource $1, \hat{R}_{1}(\theta,(1,0))$, where in each case it is assumed that the consumer population is at its population dynamical equilibrium (Mathematica notebook spelling out the algebraic details available on request).

In order to determine the behavior of mutants the same condition (A1) has to be employed. However, for mutants the right-hand side of this equation is generally not equal to one because the traits governing the mutant's functional response do not equal the traits that determine the abundance of the resources.

\section{Appendix B: Frequency-dependent selection}

In this appendix we prove that for our model, selection is frequency dependent whenever a population is behaviorally polymorphic, that is, when different genotypes show a different diet composition. In classical population genetics frequency dependence is defined as a dependence of selection coefficients on allele frequencies. In density-regulated populations a type with a positive growth rate when rare will have a zero growth rate after fixation, given the population is at its population dynamical attractor. Hence, under density regulation invasion fitness depends on frequencies by default and the classical definition of frequency dependence becomes meaningless. Following Heino et al. (1998) we therefore use the term in a slightly different way that allows to distinguish cases where the direction of evolutionary change depends on the frequency of different phenotypes within a density-regulated population. 
We refer the reader to Rueffler et al. (2006a) and especially appendix B therein for an introduction to the concept of "feedback environment" and the significance of the dimension of the feedback vector $\boldsymbol{I}$. In accordance with Heino et al. (1998) we define selection there as frequency independent when $\operatorname{dim} \boldsymbol{I}=1$ and as frequency dependent when $\operatorname{dim} \boldsymbol{I}>1$. In Rueffler et al. (2006a) we show that if evolutionary change occurs in $\boldsymbol{\alpha}, \boldsymbol{t}_{\mathrm{p}}$ and $\boldsymbol{t}_{\mathrm{m}}$ while the population is monomorphic for $\boldsymbol{e}$ and $\boldsymbol{f}$, then selection is always frequency independent, that is, $\operatorname{dim} \boldsymbol{I}=1$. On the contrary, if evolution occurs in $\boldsymbol{e}$ or $\boldsymbol{f}$, then $\operatorname{dim} \boldsymbol{I}=2$ and evolution is frequency dependent. Here we restrict ourselves to show that in behaviorally dimorphic populations necessarily $\operatorname{dim} \boldsymbol{I}>1$. This means that through the effect of diet choice selection becomes frequency dependent in cases where it would be frequency independent in the absence of diet choice.

Assume for the moment, that two different genotypes, $\theta^{1}$ and $\theta^{2}$, are present in the resident population with corresponding manipulation times $\boldsymbol{t}_{\mathrm{m}}^{1}=\left(t_{\mathrm{m} 1}^{1}, t_{\mathrm{m} 2}^{1}\right)$ and $\boldsymbol{t}_{\mathrm{m}}^{2}=\left(t_{\mathrm{m} 1}^{2}, t_{\mathrm{m} 2}^{2}\right)$, respectively. These morphological traits result in the genotype specific behavior $\boldsymbol{p}^{1}=\left(p_{1}^{1}, p_{2}^{1}\right)$ and $\boldsymbol{p}^{2}=\left(p_{1}^{2}, p_{2}^{2}\right)$, search probabilities $s^{1}$ and $s^{2}$ and densities $\hat{N}^{1}$ and $\hat{N}^{2}$, respectively. Note that superscripts refer to the different types while subscripts refer to resource specific traits. The equations for the resource equilibria (eq. 6) become

$$
\hat{R}_{1}\left(\theta^{1}, \theta^{2}\right)=\frac{b_{1}}{d_{1}+e_{1} f_{1}\left(p_{1}^{1} s^{1} \hat{N}^{1}+p_{1}^{2} s^{2} \hat{N}^{2}\right)} \text { and } \hat{R}_{2}\left(\theta^{1}, \theta^{2}\right)=\frac{b_{2}}{d_{2}+e_{2} f_{2}\left(p_{2}^{1} s^{1} \hat{N}^{1}+p_{2}^{2} s^{2} \hat{N}^{2}\right)} .
$$

In these equations $b_{i}, d_{i}, e_{i}$ and $f_{i}$ are assumed to stay constant over evolutionary time. In order to track changes in the resource equilibria while the consumer population is evolving the following two-dimensional $\boldsymbol{I}$-vector has to be followed: $\boldsymbol{I}=\left(\sum_{j=1}^{2} p_{1}^{j} s^{j} \hat{N}^{j}, \sum_{j=1}^{2} p_{2}^{j} s^{j} \hat{N}^{j}\right)$. Note, that when $\boldsymbol{p}^{1}=\boldsymbol{p}^{2}$ this $\boldsymbol{I}$-vector simplifies to a the scalar $\boldsymbol{I}=\sum_{j=1}^{2} s^{j} \hat{N}^{j}$. The identical result holds when the population is dimorphic in $\boldsymbol{t}_{\mathrm{p}}$ or $\boldsymbol{\alpha}$. In populations dimorphic in $\boldsymbol{f}$ the environmental condition is given by $\boldsymbol{I}=\left(\sum_{j=1}^{2} f_{1}^{j} p_{1}^{j} s^{j} \hat{N}^{j}, \sum_{j=1}^{2} f_{2}^{j} p_{2}^{j} s^{j} \hat{N}^{j}\right)$. The result for a population dimorphic in $\boldsymbol{e}$ is analogous.

\section{Appendix C: Individual-based simulations}

In this appendix we sketch the algorithm used for the individual-based simulations. Within a generation, we start by determining the within-year resource equilibria. To this end we use Euler's forward method for solving the ordinary differential equations describing the resource dynamics (eq. 5). In each step we advance the solution through an interval of size $h=2 * 10^{-3}$. After each increment the optimal consumer behavior is determined for each genotype present in the population. Based on the resource densities and consumer behavior found after 1000 steps in the Euler algorithm the deterministic number of offspring $R_{0}$ for each consumer genotype $i$ is calculated as $N_{t+1}^{i} / N_{t}^{i}$ (according to eq. 1). The actual offspring number per individual is then determined by drawing a random number from a poisson distribution with mean $R_{0}$. Mutations occur with probability $\mu=1 * 10^{-4}$ per offspring. Mutant phenotypes are determined by drawing a random 
number from a normal distribution, truncated to [0.01,0.99], with the mother's trait value as mean and standard deviation $\sigma=0.02$. All simulations are initialized with the resident population at its ecological equilibrium, which ranges from 9000 to 10000 individuals, depending on the trait value. 


\section{REFERENCES}

Abrams, P. A. 1986. Character displacement and niche shift analyzed using consumer-resource models of competition. Theoretical Population Biology 29:107-160.

Abrams, P. A. 1999. The adaptive dynamics of consumer choice. The American Naturalist 153:8397.

Abrams, P. A. 2006. The prerequisites for and liklihood of generalist-specialist coexistence. The American Naturalist 167:329-342.

Abrams, P. A. and Matsuda, H. 2003. Population dynamical consequences of reduced predator switching at low total prey densities. Population Ecology 45:175-185.

Abrams, P. A. and Matsuda, H. 2004. Consequences of behavioral dynamics for the population dynamics of predator-prey systems with switching. Population Ecology 46:13-25.

Abrams, P. A., Matsuda, H., and Harada, Y. 1993. Evolutionary unstable fitness maxima and stable fitness minima of continuous traits. Evolutionary Ecology 7:465-487.

Bateson, P., 1988. The active role of behavior in evolution. Pages 191-207 in M.-W. Ho and S. W. Fox, eds. Processes and Metaphors in Evolution. Wiley, Chichester.

Bolnick, D. I. and Doebeli, M. 2003. Sexual dimorphism and adaptive speciation: Two sides of the same ecological coin. Evolution 57:2433-2449.

Brown, J. S. 1990. Habitat selection as an evolutionary game. Evolution 44:732-746.

Brown, J. S. 1996. Coevolution and community organization in three habitats. Oikos 75:193-206.

Case, T. J., 2000. An Illustrated Guide to Theoretical Ecology. Oxford University Press.

Christiansen, F. B. 1991. On conditions for evolutionary stability for a continuously varying character. The American Naturalist 138:37-50.

Eshel, I. 1983. Evolutionary and continuous stability. Journal of Theoretical Biology 103:99-111.

Eshel, I. and Motro, U. 1981. Kin selection and strong evolutionary stability of mutual help. Theoretical Population Biology 19:420-433.

Geritz, S. A. H. and Kisdi, E. 2004. On the mechanistic underpinning of discrete-time population models with complex dynamics. Journal of Theoretical Biology 228:261-269.

Geritz, S. A. H., Kisdi, E., Meszéna, G., and Metz, J. A. J. 1998. Evolutionarily singular strategies and the adaptive growth and branching of the evolutionary tree. Evolutionary Ecology $12: 35-57$. 
Geritz, S. A. H., van der Meijden, E., and Metz, J. A. J. 1999. Evolutionary dynamics of seed size and seedling competitive ability. Theoretical Population Biology 55:324-343.

Giraldeau, L.-A. and Caraco, T., 2000. Social Foraging Theory. Monographs in Behavior and Ecology. Princeton University Press, Princeton, NJ.

Heino, M., Metz, J. A. J., and Kaitala, V. 1998. The enigma of frequency-dependent selection. Trends in Ecology and Evolution 13:367-370.

Krebs, J. R., Erichsen, J. T., Webber, M. I., and Charnov, E. L. 1977. Optimal prey selection by the great tit (parus major). Animal Behaviour 25:30-38.

Lawlor, L. R. and Maynard Smith, J. 1976. The coevolution and stability of competing species. The American Naturalist 110:79-99.

Levins, R. 1962. Theory of fitness in a heterogeneous environment. i. the fitness set and the adaptive function. The American Naturalist 96:361-373.

Lundberg, S. and Stenseth, N. C. 1985. Coevolution of competing species: Ecological character displacement. Theoretical Population Biology 27:105-119.

Maynard Smith, J., 1982. Evolution and the Theory of Games. Cambridge University Press, Cambridge, U. K.

Metz, J. A. J., in press. Chapter in J. A. J. Metz and U. Dieckmann, eds. Elements of Adaptive Dynamics. Cambridge University Press, Cambridge, U.K.

Metz, J. A. J., Geritz, S. A. H., Meszéna, G., Jacobs, F. J. A., and Van Heerwaarden, J. S., 1996. Adaptive dynamics: A geometrical study of the consequences of nearly faithful reproduction. Pages 183-231 in S. J. van Strien and S. Verduyn Lunel, eds. Stochastic and spatial structures of dynamical systems, Proceedings of the Royal Dutch Academy of Science. North Holland, Dordrecht, Netherlands; available at http://www.iiasa.ac.at/Research/ADN/Series.html.

Metz, J. A. J., Nisbet, R. M., and Geritz, S. A. H. 1992. How should we define 'fitness' for general ecological scenarios? Trends in Ecology and Evolution 7:198-202.

Plotkin, H. C., ed., 1988. The Role of Behavior in Evolution. The MIT Press, Cambridge, Massachusetts.

Rosenzweig, M. L. 1981. A theory of habitat selection. Ecology 62:327-335.

Rosenzweig, M. L. 1987. Habitat selection as a source of biological diversity. Evolutionary Ecology 1:315-330.

Rueffler, C., Van Dooren, T. J. M., and Metz, J. A. J. 2004. Adaptive walks on changing landscapes: Levins' approached extended. Theoretical Population Biology 65:165-178. 
Rueffler, C., Van Dooren, T. J. M., and Metz, J. A. J. 2006a. The evolution of resource specialization through frequency-dependent and frequency-independent mechanisms. The American Naturalist 167:81-93.

Rueffler, C. Van Dooren, T. J. M., Leimar, O., and Abrams, P. A. 2006b. Disruptive selection and then what? Trends in Ecology and Evolution 21:238-245.

Schaffer, W. M. 1981. Ecological abstraction: The consequences of reduced dimensionality in ecological models. Ecological Monographs 51:383-401.

Schoener, T. 1978. Effects of density-restricted food encounter on some single-level competition models. Theoretical Population Biology 13:365-381.

Stenseth, N. C. 1984. Evolutionary stable strategies in food selection models with fitness sets. Journal of Theoretical Biology 109:489-499.

Stephens, D. W. and Krebs, J. R., 1986. Foraging Theory. Princeton University Press, Princeton, New Jersey.

Svanbäck, R. and Bolnick, D. I. 2005. Intraspecific competition affects the strength of individual specialization: an optimal diet theory method. Evolutionary Ecology Research 7:993-1012.

Van Dooren, T. J. M., in press. Chapter in J. A. J. Metz and U. Dieckmann, eds. Elements of Adaptive Dynamics. Cambridge University Press, Cambridge, U.K.

Van Dooren, T. J. M., Demon, I., and Durinx, M. 2004. Sexual dimorphism or evolutionary branching? Evolutionary Ecology Research 6:857-871.

Vincent, T. L. S., Scheel, D., Brown, J. S., and Vincent, T. L. 1996. Trade-offs and coexistence in consumer-resource models: It all depends on what and where you eat. The American Naturalist 148:1038-1058.

Waddington, C. H., 1975. The Evolution of an Evolutionist. Edinburgh University Press.

Waxman, D. and Gavrilets, S. 2005. 20 questions on adaptive dynamics. Journal of Evolutionary Biology 18:1139-1154.

Wcislo, W. T. 1989. Behavioural environments and evolutionary change. Annual Review in Ecology and Systematics 20:137-169.

Wilson, D. S. and Turelli, M. 1986. Stable underdominance and the evolutionary invasion of empty niches. The American Naturalist 127:835-850. 
Table 1: Notation. The index $i$ refers to one out of two possible resources.

\begin{tabular}{ll}
\hline Symbol & \multicolumn{1}{c}{ Definition } \\
\hline$\alpha_{i}$ & Conversion efficiency of consumed resource into offspring \\
$b_{i}$ & Constant resource influx \\
$C_{i}$ & Consumer's functional response \\
$d_{i}$ & Death rate of resource \\
$e_{i}$ & Consumer's search efficiency [area/time step] \\
$f_{i}$ & Capture probability for an attacked resource item \\
$N_{t}$ & Consumer population density at time step $t$ \\
$p_{i}$ & Consumer's probability of attack upon encounter with resource \\
$R_{i}$ & Density of resources [1/area] \\
$t_{\mathrm{m} i}$ & Manipulation time \\
$t_{\mathrm{p} i}$ & (needed for treatment of an already capture resource item) \\
$s$ & Pursuit time (needed to catch an attacked resource item) \\
$\theta$ & Search probability (fraction of time spent searching for resources) \\
& Specialization coefficient $\in[0,1]$ \\
$w$ & (determines location on the trade-off curve) \\
$z$ & Invasion fitness \\
\hline
\end{tabular}

Table 2: Overview of traits considered evolvable. Each parameter stands for a vector of two traits coupled by trade-off.

\begin{tabular}{lc}
\hline Trait & Behavioral switch \\
\hline Conversion efficiency, $\boldsymbol{\alpha}$ & yes \\
Search efficiency, $\boldsymbol{e}$ & no \\
Capture probability, $\boldsymbol{f}$ & yes (if $\boldsymbol{t}_{\mathrm{m}}$ not negligible) \\
Manipulation time, $\boldsymbol{t}_{\mathrm{m}}$ & yes \\
Pursuit time, $\boldsymbol{t}_{\mathrm{p}}$ & yes \\
\hline
\end{tabular}

Fig. 1.- Trade-off in capture probability $\boldsymbol{f}$ (a) and manipulation time $\boldsymbol{t}_{\mathrm{m}}$ (b). The number next to each curve is the parameter $z$ determining the strength of the trade-off. For capture probability a weak trade-off $(z>1)$ corresponds to a concave curve while for manipulation time a weak trade-off corresponds to a convex curve. The trade-off curve is parameterized such that $\theta=0$ corresponds to a specialist for resource 1 and $\theta=1$ corresponds to a specialist for resource 2 . Therefore the two trade-off curves are parameterized in opposite direction. Filled circles half way on the trade-off curve correspond to the generalist with $\theta=0.5$. Other parameter values: (a) $\boldsymbol{f}_{\max }=(1,1)$, (b) $\boldsymbol{t}_{\operatorname{mmax}}=(1,1), \boldsymbol{t}_{\mathrm{mmin}}=(0.5,0.5)$. 
Fig. 2.- Bifurcation diagram of singular strategies with bifurcation parameter $z$, the parameter determining the strength of the trade-off curve. Phenotypes are represented by the specialization coefficient $\theta$. Arrows give the direction of evolutionary change. Hatched areas indicate parameter combinations corresponding to non-viable populations. White areas indicate opportunistic foraging behavior while gray areas indicate selective foraging behavior. Results for capture probability $\boldsymbol{f}$ (a) and manipulation time $\boldsymbol{t}_{\mathrm{m}}$ (b). Results for pursuit time $\boldsymbol{t}_{\mathrm{p}}$ and conversion efficiency $\boldsymbol{\alpha}$ are qualitatively identical to those in (b). The solid vertical black line in (b) indicates that for $z=1$ all morphologies corresponding to opportunistic behavior are selectively neutral. Other parameter values: (a) $\boldsymbol{t}_{\mathrm{m}}=(0.5,0.5), \boldsymbol{f}_{\max }=(1,1)$, (b) $\boldsymbol{t}_{\operatorname{mmin}}=(0.5,0.5), \boldsymbol{t}_{\operatorname{mmax}}=(1,1), \boldsymbol{f}=(1,1),(\mathrm{a}, \mathrm{b})$ $\boldsymbol{\alpha}=(1,1), \boldsymbol{e}=(0.05,0.05), \boldsymbol{t}_{\mathrm{p}}=(0.1,0.1), \boldsymbol{b}=(5000,5000), \boldsymbol{d}=(0.1,0.1)$.

Fig. 3.- Pairwise invadability plots (PIPs) for capture probability $\boldsymbol{f}(\mathrm{a}, \mathrm{b})$ and manipulation time $\boldsymbol{t}_{\mathrm{m}}(\mathrm{c}, \mathrm{d})$. White areas indicate combinations of mutants and residents where the mutant is able to invade while gray areas correspond to combinations where the mutant is doomed to extinction. For each trait one PIP corresponds to a strong trade-off with $z=0.8$ (a, c) while the other corresponds to a weak trade-off with $z=1.2(\mathrm{~b}, \mathrm{~d})$. Diet choice of residents, $\boldsymbol{p}=\left(p_{1}, p_{2}\right)$, is indicated at the underbraces at the $x$-axes. Black solid lines are mutant choice boundaries that indicate changes in the diet choice of mutants, $\boldsymbol{p}^{\prime}=\left(p_{1}^{\prime}, p_{2}^{\prime}\right)$. Other parameter values as in figure 2 .

Fig. 4. - Evolution in dimorphic populations for capture probability $\boldsymbol{f}$ (a, b) and manipulation time $\boldsymbol{t}_{\mathrm{m}}$ (c, d) for strong trade-offs with $z=0.8$ (a, c) and weak trade-offs with $z=1,2$ (b) and $z=1.5$ (d). Each axis gives the value of the specialization coefficient $\theta$ of one consumer type. White areas correspond to phenotype combinations that can coexist in a protected dimorphism. Diet choice at dimorphic population dynamical equilibrium for each type is indicated by the underbraces at each axis. Arrows indicate the direction of selection and dots indicate evolutionary stable endpoints. Thick lines correspond to stable isoclines while dashed lines correspond to a change in the diet composition of one of the two types. Other parameter values as in figure 2 except for $\boldsymbol{\alpha}=(0.92,0.92)$ in $(\mathrm{d})$.

Fig. 5.- Individual-based simulations of trait distributions for capture probability $f(a)$ and manipulation time $\boldsymbol{t}_{\mathrm{m}}(\mathrm{b}, \mathrm{c})$. Different tones of gray indicate behavior: black corresponds to selectors for resource 1, dark gray to opportunists and light gray to selectors for resource 2 . The populations in (a) and (b) are initially monomorphic characterized by (a) $\theta=0.35$ and (b) $\theta=0.45$. The population in (c) consists of monomorphic population characterized by $\theta=0.25$ to which 10 immigrants, characterized by $\theta=0.15$, are added. Parameter values as in figure 4. See appendix C for further details. 
Fig. 6. - PIPs for capture probability $f$ for different values of the accuracy parameter $a$ (in columns) and different curvatures for the trade-off, as determined by $z$ (in rows). Figures in the first column show the probability of attack of a resident consumer upon encounter with an item of resource 1 ( $p_{1}$, on the $y$-axis) as a function of the specialization coefficient $\theta$ of the resident ( $x$-axis) for $a=3, a=10$ and $a=\infty$ (darker curves correspond to higher values of $a$ ). Curves for $p_{2}$ are given by the mirror image of each curve around $\theta=0.5$. Switch curves in the first figure in a row and PIPs in the same row that are generated with the same value of $a$-value are shown in same shade of gray. Note that the sharp drop-off in $p_{1}$ occurs for higher values of $\theta$ with higher values of $z$. Other parameter values as in figure 2 .

Fig. 7. - Effect of foraging inaccuracy on the evolution of manipulation time $\boldsymbol{t}_{\mathrm{m}}$ in monomorphic $(\mathrm{a}, \mathrm{b}, \mathrm{d})$ and dimorphic populations (c,e,f). Figure (a) shows an example of a PIP without foraging inaccuracy $(a=\infty)$. This PIP can be generated from two different parameter combinations: (i) $z=0.56, \boldsymbol{t}_{\mathrm{p}}=(0.05,0.05)$ and (ii) $z=0.8, \boldsymbol{t}_{\mathrm{p}}=(0.1,0.1)$. Figure (b) and (d) illustrate the the effect of foraging inaccuracy $(a=50)$ for each of these parameter combinations. For the first set of parameters the degenerate singular points disappear altogether (fig. b). As a consequence two very similar resident types (close to the main diagonal) can never coexist (fig. c). For the second set of parameters the degenerate singular points unfold into an evolutionary repellor and a CSS (fig d). In this case coexistence of two types close to the main diagonal becomes possible (fig. e). However, evolutionary isoclines appear showing that similar resident types experience convergent selection such that a dimorphic population collapses to become monomorphic again (fig. e \& f). Other parameter values as in figure 2 . 

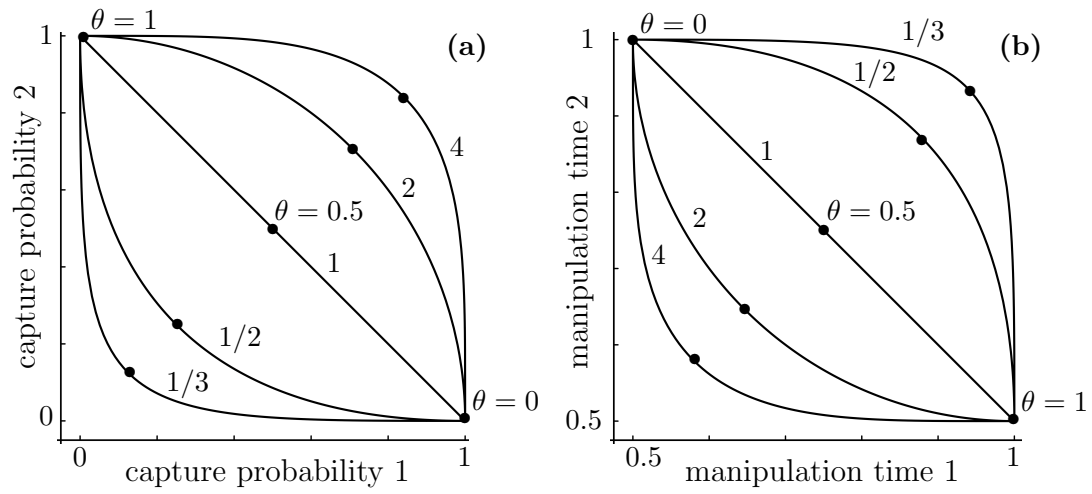

Figure 1 
(a) Capture Probability $f$

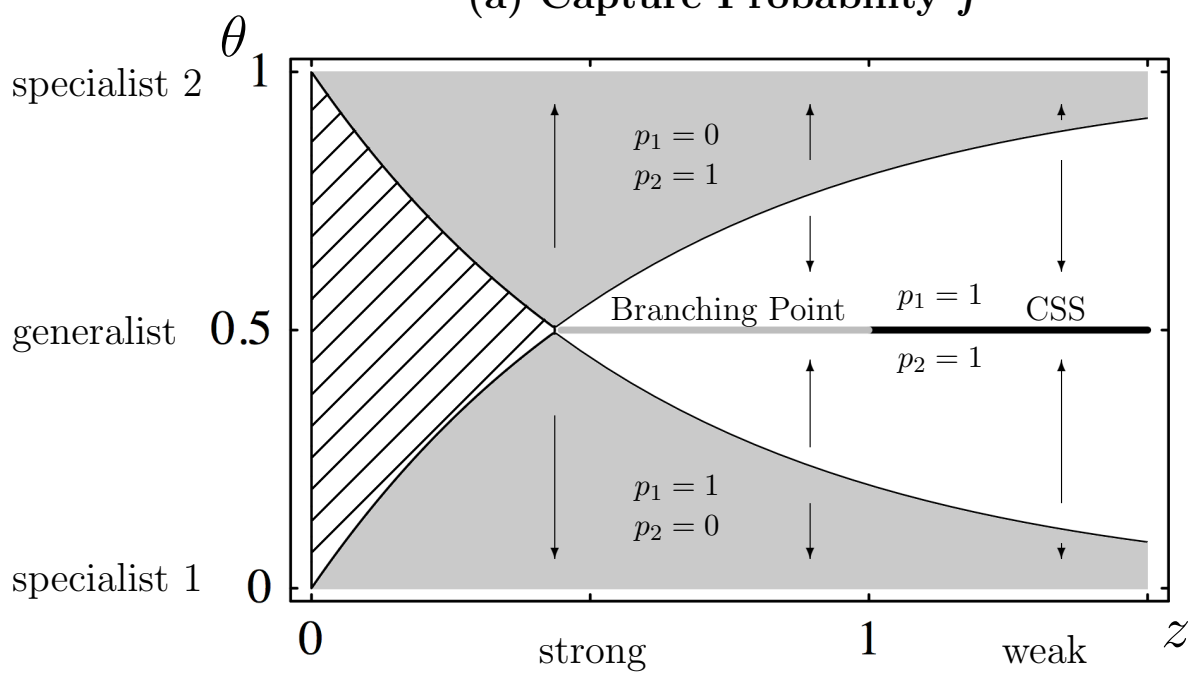

(b) Manipulation Time $t_{\mathrm{m}}$

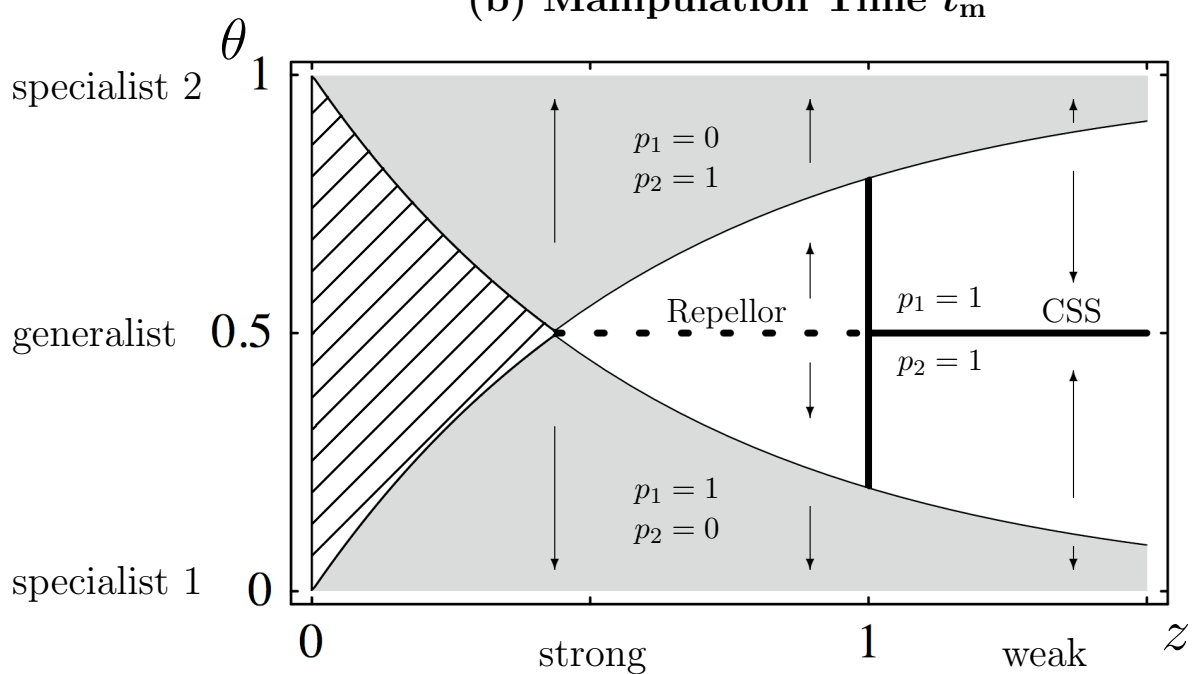

Figure 2 
strong trade-off
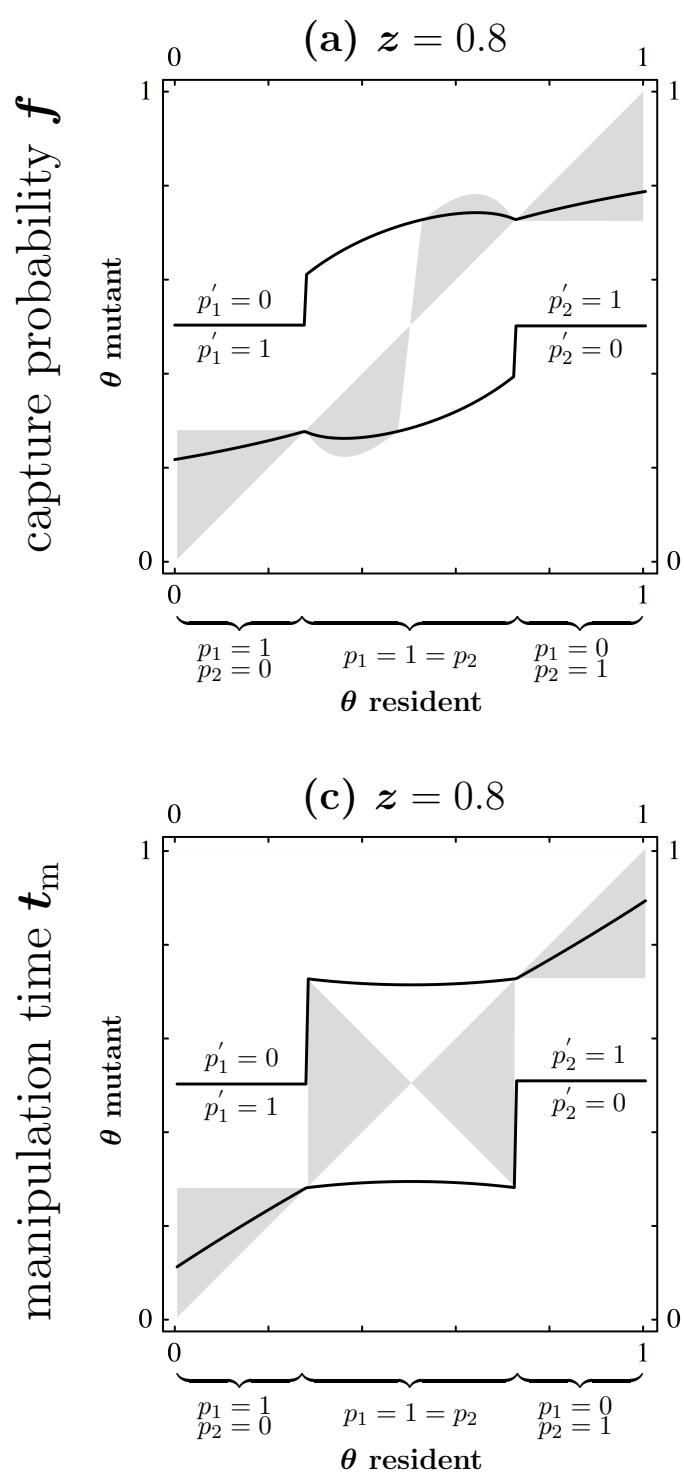

weak trade-off

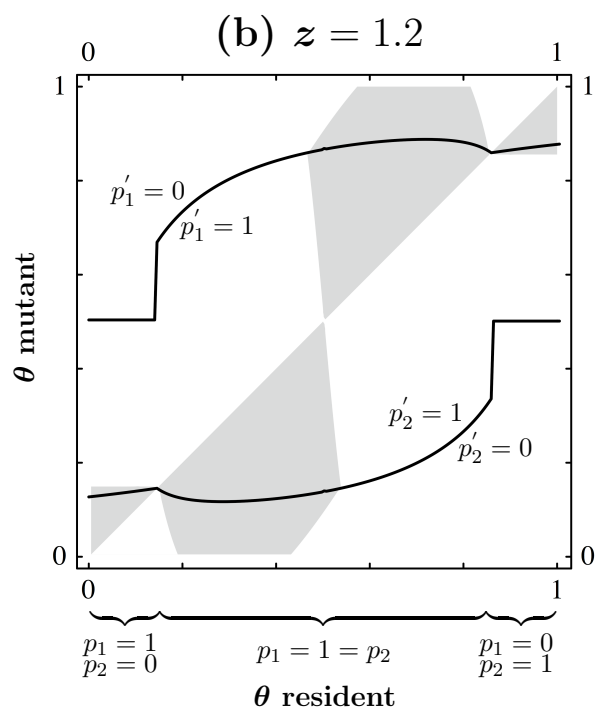

(d) $z=1.2$

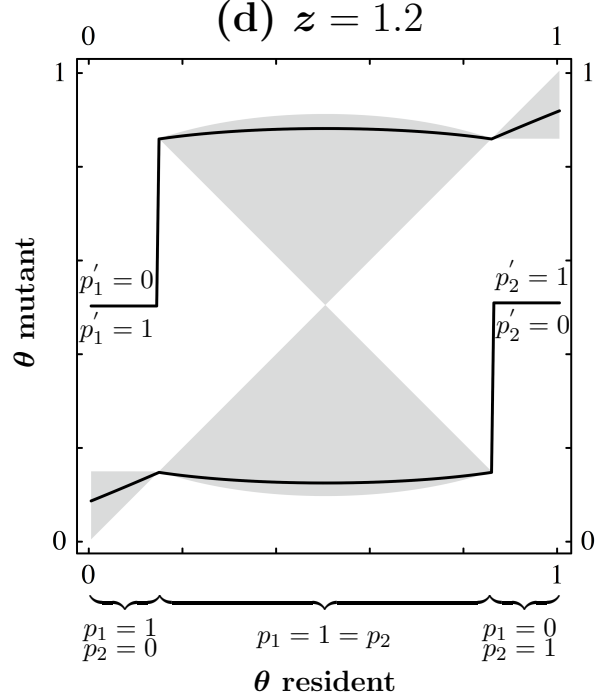

Figure 3 


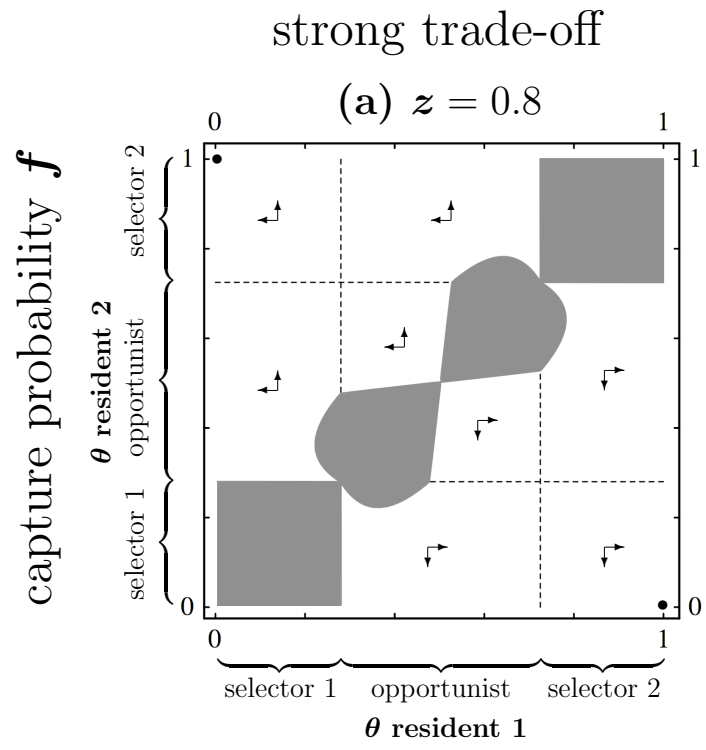

weak trade-off
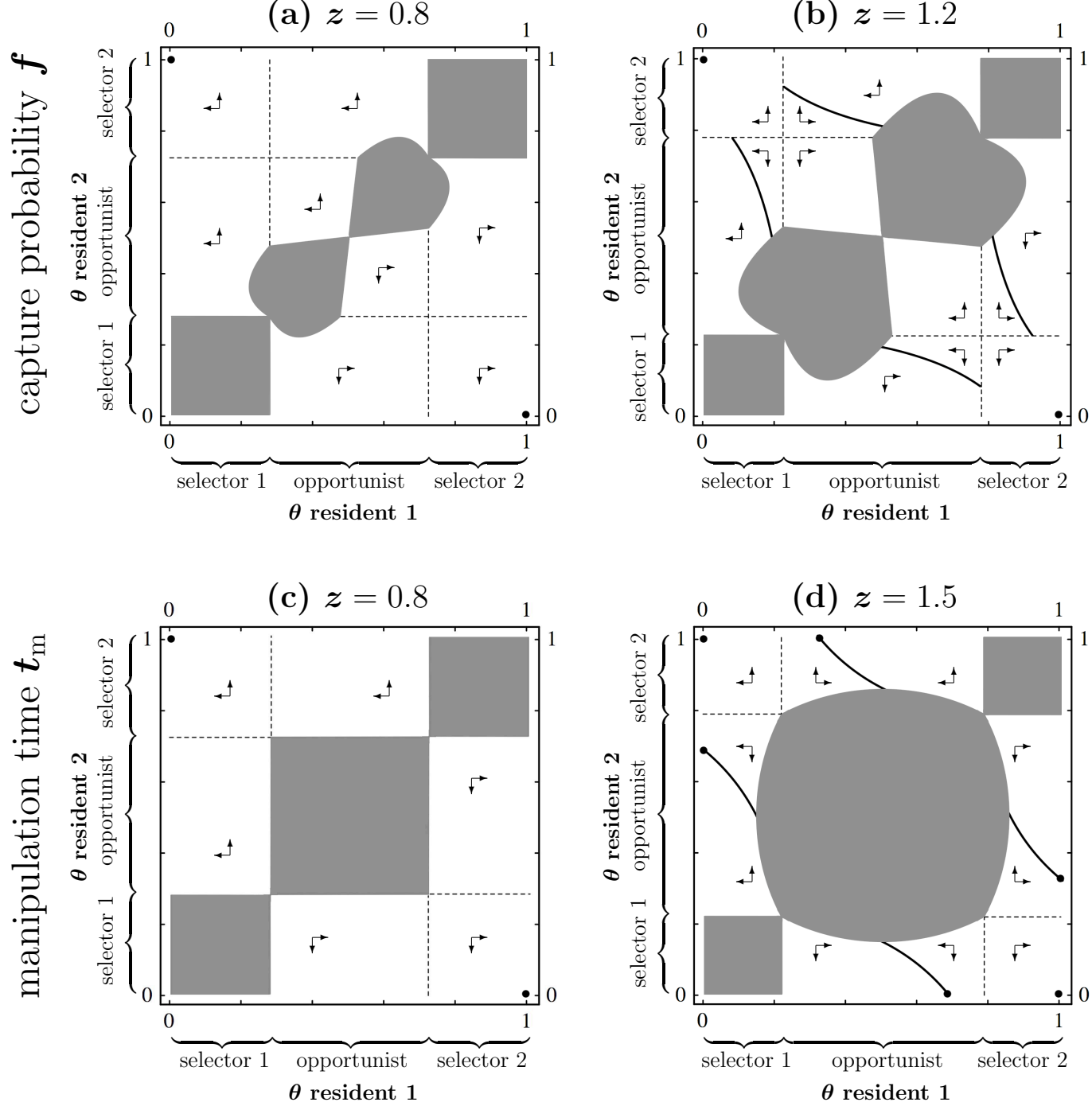

Figure 4 
(a) $\boldsymbol{f}, z=0.8$

(b) $\boldsymbol{t}_{\mathrm{m}}, z=0.8$

(c) $\boldsymbol{t}_{\mathrm{m}}, z=1.5$
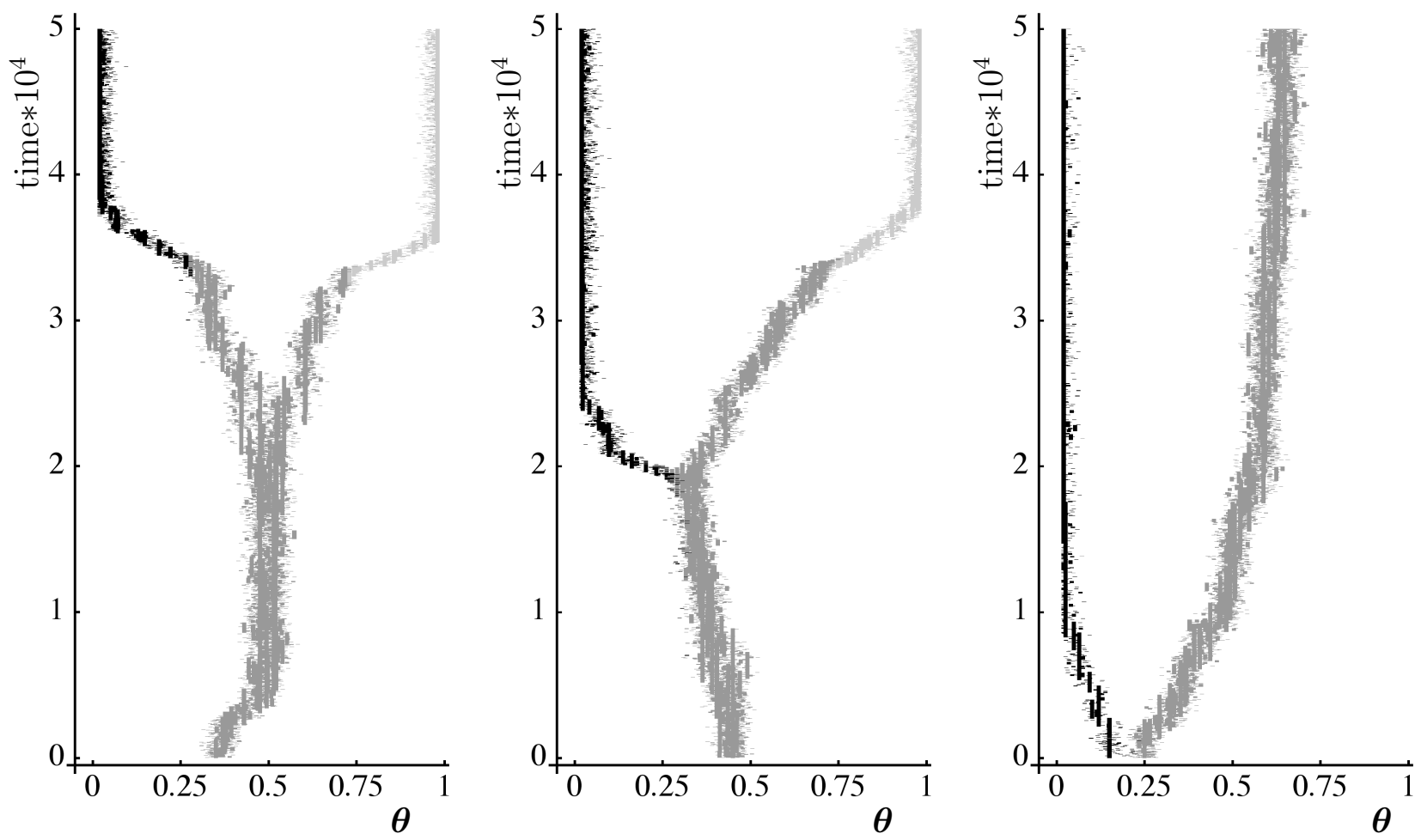


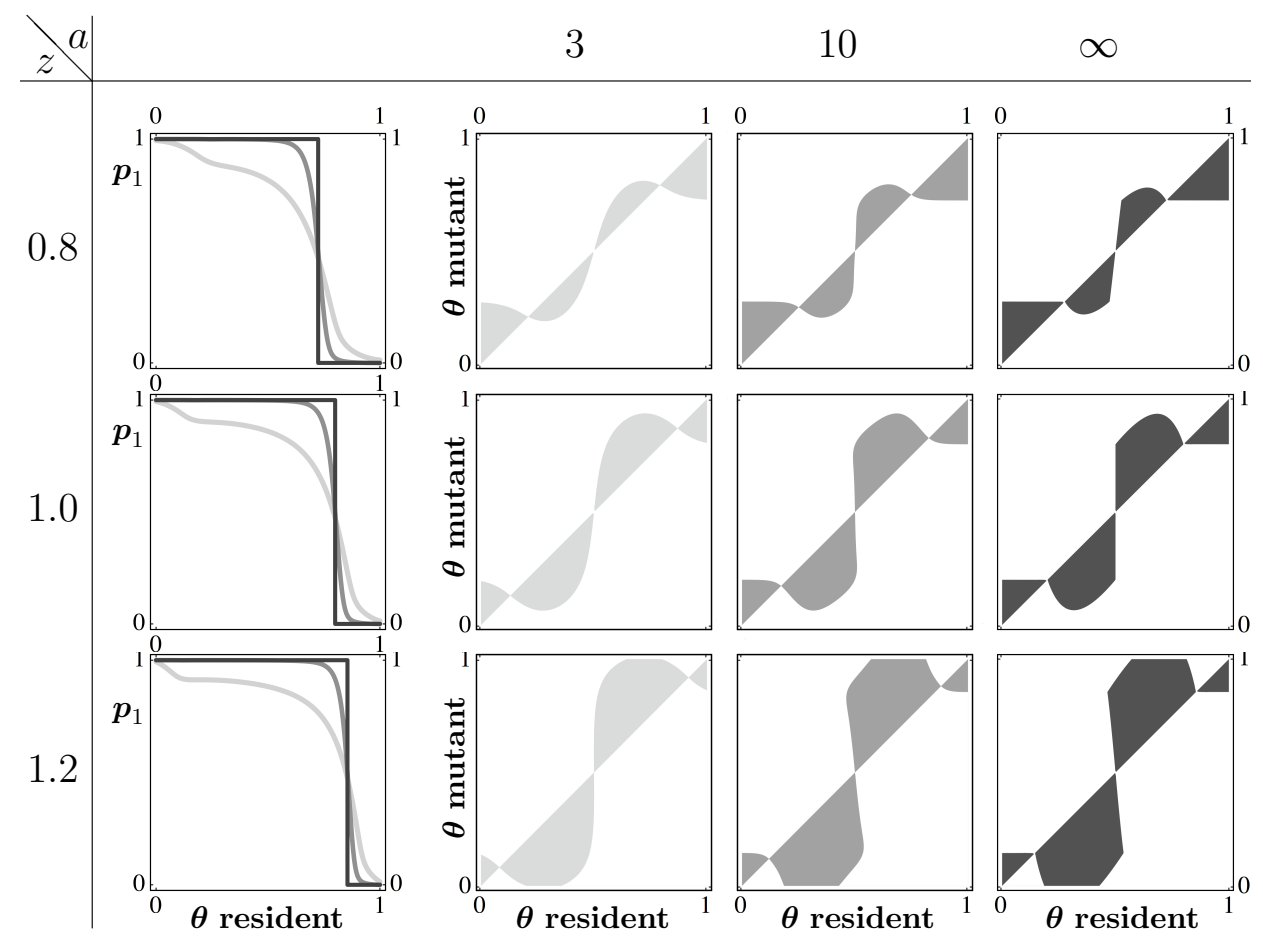

Figure 6 


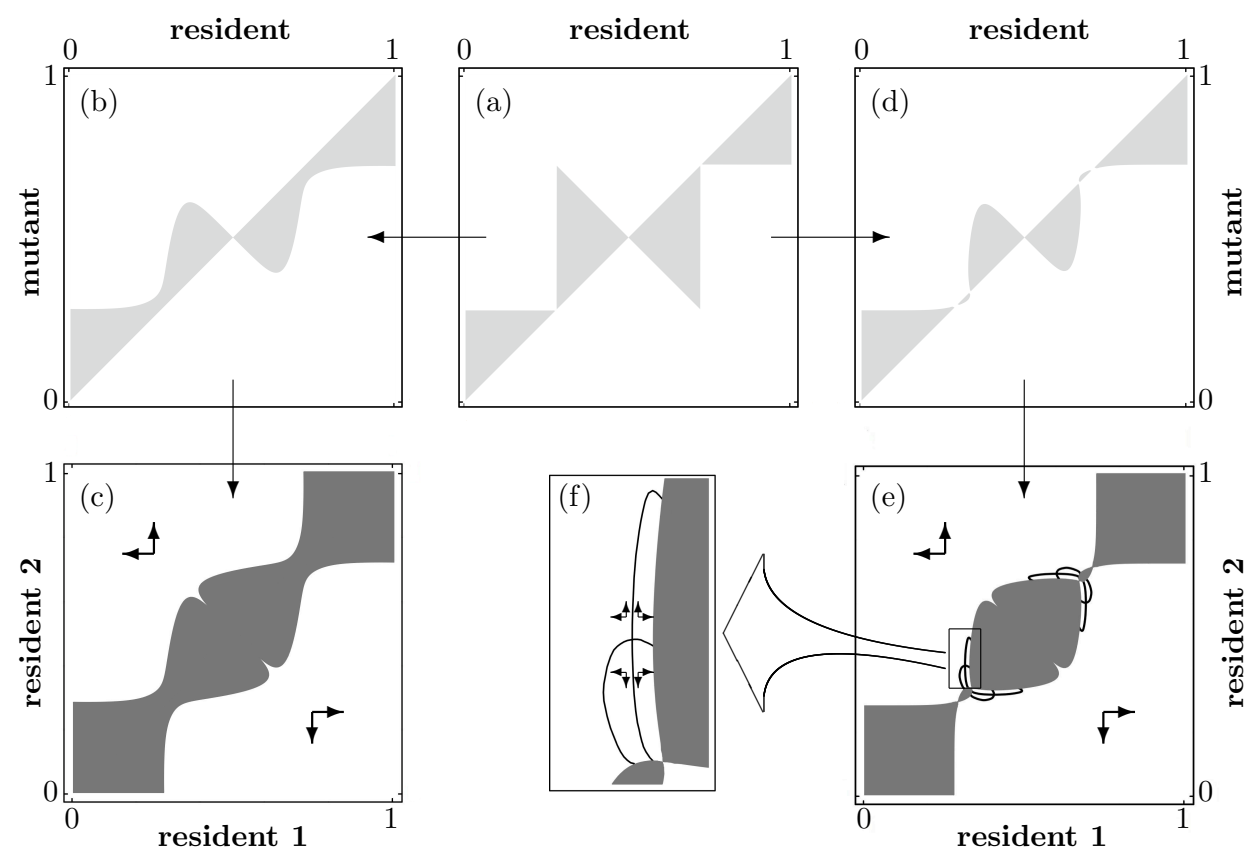

Figure 7 